\title{
Carbonaceous aerosols in China: top-down constraints on primary sources and estimation of secondary contribution
}

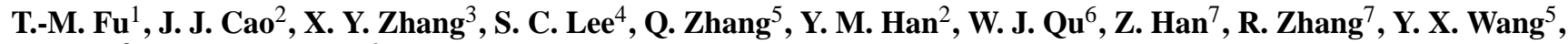 \\ D. Chen ${ }^{8}$, and D. K. Henze ${ }^{9}$ \\ ${ }^{1}$ Department of Atmospheric and Oceanic Sciences and Laboratory for Climate and Ocean-Atmosphere Studies, School of \\ Physics, Peking University, Beijing, China \\ ${ }^{2}$ State Key Laboratory of Loess and Quaternary Geology, Institute of Earth Environment, Chinese Academy of Sciences, \\ Xi' an, China \\ ${ }^{3}$ Key Laboratory for Atmospheric Chemistry, Centre for Atmosphere Watch \& Services of CMA, Chinese Academy of \\ Meteorological Sciences, Beijing, China \\ ${ }^{4}$ Research Centre of Urban Environmental Technology and Management, Department of Civil and Structural Engineering, \\ Hong Kong Polytechnic University, Kowloon, Hong Kong \\ ${ }^{5}$ Ministry of Education Key Laboratory for Earth System Modeling, Center for Earth System Science, Institute for Global \\ Change Studies, Tsinghua University, Beijing, China \\ ${ }^{6}$ Physical Oceanography Laboratory, Ocean-Atmosphere Interaction and Climate Laboratory, Ocean University of China, \\ Qingdao, China \\ ${ }^{7}$ Key Laboratory of Regional Climate-Environment Research for Temperate East Asia, Institute of Atmospheric Physics, \\ Chinese Academy of Sciences, Beijing, China \\ ${ }^{8}$ Department of Atmospheric \& Oceanic Sciences, University of California, Los Angeles, Los Angeles, USA \\ ${ }^{9}$ Department of Mechanical Engineering, University of Colorado at Boulder, Boulder, USA \\ Correspondence to: T.-M. Fu (tmfu@ pku.edu.cn)
}

Received: 15 August 2011 - Published in Atmos. Chem. Phys. Discuss.: 20 October 2011

Revised: 2 February 2012 - Accepted: 6 February 2012 - Published: 14 March 2012

\begin{abstract}
We simulated elemental carbon (EC) and organic carbon (OC) aerosols in China and compared model results to surface measurements at Chinese rural and background sites, with the goal of deriving "top-down" emission estimates of EC and OC, as well as better quantifying the secondary sources of OC. We included in the model state-of-the-science Chinese "bottom-up" emission inventories for $\mathrm{EC}\left(1.92 \mathrm{TgC} \mathrm{yr}^{-1}\right)$ and $\mathrm{OC}\left(3.95 \mathrm{TgC} \mathrm{yr}^{-1}\right)$, as well as updated secondary $\mathrm{OC}$ formation pathways. The average simulated annual mean EC concentration at rural and background sites was $1.1 \mu \mathrm{gC} \mathrm{m}^{-3}, 56 \%$ lower than the observed $2.5 \mu \mathrm{gC} \mathrm{m}^{-3}$. The average simulated annual mean OC concentration at rural and background sites was $3.4 \mu \mathrm{gC} \mathrm{m}{ }^{-3}$, $76 \%$ lower than the observed $14 \mu \mathrm{gC} \mathrm{m}{ }^{-3}$. Multiple regression to fit surface monthly mean EC observations at rural and background sites yielded the best estimate of Chinese EC source of $3.05 \pm 0.78 \mathrm{TgC} \mathrm{yr}^{-1}$. Based on the topdown EC emission estimate and observed seasonal primary OC/EC ratios, we estimated Chinese OC emissions to be
\end{abstract}

$6.67 \pm 1.30{\mathrm{TgC} \mathrm{yr}^{-1}}^{-1}$ Using these top-down estimates, the simulated average annual mean EC concentration at rural and background sites was significantly improved to $1.9 \mu \mathrm{\mu C} \mathrm{m} \mathrm{m}^{-3}$. However, the model still significantly underestimated observed OC in all seasons (simulated average annual mean OC at rural and background sites was $5.4 \mu \mathrm{gC} \mathrm{m}^{-3}$ ), with little skill in capturing the spatiotemporal variability. Secondary formation accounts for $21 \%$ of Chinese annual mean surface OC in the model, with isoprene being the most important precursor. In summer, as high as $62 \%$ of the observed surface OC may be due to secondary formation in eastern China. Our analysis points to four shortcomings in the current bottom-up inventories of Chinese carbonaceous aerosols: (1) the anthropogenic source is underestimated on a national scale, particularly for OC; (2) the spatiotemporal distributions of emissions are misrepresented; (3) there is a missing source in western China, likely associated with the use of biofuels or other low-quality fuels for heating; and (4) sources in fall are not well represented, either because the seasonal shifting of 
emissions and/or secondary formation are poorly captured or because specific fall emission events are missing. In addition, secondary production of OC in China is severely underestimated. More regional measurements with better spatiotemporal coverage are needed to resolve these shortcomings.

\section{Introduction}

Carbonaceous aerosols, including elemental carbon aerosol (EC) and organic carbon aerosol (OC), are important components of atmospheric particulate matter, affecting air quality and climate. EC, alternatively referred to as black carbon aerosol (BC), is emitted directly into the atmosphere from combustion associated with anthropogenic activities and biomass burning. OC can either be emitted as primary particles from combustion or produced in the atmosphere as part of secondary organic aerosol (SOA) from gaseous organic compounds. In this study, we use surface observations to constrain the primary and secondary sources of carbonaceous aerosols in China.

Estimates of Chinese EC and OC emissions are highly uncertain (Streets et al., 2003a; Ohara et al., 2007; Zhang et al., 2009; Lei et al., 2011; Lu et al., 2011). Emission inventories are traditionally constructed from the "bottom up" approach based on activity data and emission factors. However, accurate and detailed activity statistics are often unavailable in China. Emission factors representative of local conditions are scarcely measured, and the values are highly variable depending on fuel type and combustion conditions. Some emission factors differ by orders of magnitude from western values (Streets et al., 2001, 2003a; Bond et al., 2004; Cao et al., 2006; Ohara et al., 2007; Zhang et al., 2009; Lei et al., 2011; Qin and Xie, 2011; and references therein). As a result, there are large uncertainties associated with Chinese emissions from small industries (such as coke and brick production), residential combustion, and transportation (Zhang et al., 2009; Lu et al., 2011); all three activities are important sources of carbonaceous aerosols.

Only a few studies have examined the validity of Chinese carbonaceous aerosol emission inventories by applying them to numerical models and comparing results against observations. Hakami et al. (2005) used an adjoint model and surface measurements in and around Korea and Japan in spring 2001 to test the anthropogenic and biomass burning EC emission inventories developed by Streets et al. (2003a, b). They concluded that while the estimated emission totals for East Asia were consistent with observations, there were sizable sub-regional differences. In particular, anthropogenic emissions from northeastern China and Japan were underestimated, while those from southeastern China were overestimated. Z. Han et al. (2008) also used the anthropogenic and biomass burning emission inventories developed by Streets et al. (2003a, b) to simulate summertime primary and secondary carbonaceous aerosols in eastern China. They compared model results against surface observations at 14 urban and 3 rural sites in summer 2003 and found both EC and $\mathrm{OC}$ to be underestimated at almost all sites. Matsui et al. (2009) calculated the anthropogenic EC and OC emissions for northern China for the year 2004, applied their inventory in a regional model, and compared simulated concentrations to observations in Beijing in summer 2006. They found that the model overestimated EC at one urban site and one suburban site in Beijing, as well as overestimated OC at the suburban site. Kondo et al. (2011) measured yearround $\mathrm{BC}$ concentrations on a remote island in East China Sea, downwind from China. They selectively analysed data strongly impacted by transport from China (mostly in winter and spring) and estimated Chinese annual anthropogenic BC emission to be $1.92 \mathrm{TgC} \mathrm{yr}^{-1}$, similar to the $1.81 \mathrm{TgC} \mathrm{yr}^{-1}$ estimated by Zhang et al. (2009). Each of these previous studies was limited to a particular location and/or a particular season. Koch et al. (2009) analyzed results from 17 global models using different emission inventories. They found that 16 out of the 17 models severely underestimated the observed annual mean surface EC concentrations in China. To the best of our knowledge, no detailed evaluation has been conducted on Chinese carbonaceous aerosol emission estimates at the national scale for all seasons.

Very little is known about the secondary sources of OC in China. Zhang et al. (2005) measured EC and OC at a coastal site in eastern China from June to December 2003 and at an inland site in northeastern China from June to August 2003. Based on trajectory analyses and the minimum OC/EC ratios observed in clean air masses, they found that $37-57 \%$ of the total OC could be attributed to secondary sources. Cao et al. (2007) measured EC and OC concentrations in 14 Chinese cities in January and July 2003. They estimated that $40 \%$ of Chinese urban surface OC is secondary. X. Y. Zhang et al. (2008) analyzed year-round EC and OC measurements at 18 urban, rural, and background sites. They estimated that secondary OC (SOC) accounts for $48-62 \%$ and $53-80 \%$ of urban and rural annual surface OC, respectively, based on the observed annual minimum OC/EC ratios at each site. $X$. Wang et al. (2009) simulated SOA in a regional chemistry model and found that $55 \%$ to $65 \%$ of SOA came from aromatic volatile organic compounds (VOC) in the Pearl River Delta region in spring. Also using a regional chemistry model, Liu et al. (2010) concluded that SOC produced from biogenic and anthropogenic VOC accounted for $20 \%$ of annual mean surface OC in eastern China. Neither X. Wang et al. (2009) nor Liu et al. (2010) compared model results with observations. Z. Han et al. (2008) simulated summertime SOC produced by biogenic VOC in eastern China. They found that SOC made up $50 \%$ of the simulated surface $\mathrm{OC}$ in the forested areas of southeastern, southwestern and northeastern China in summer, although their simulated total OC concentrations are $40 \%$ lower than observed values. Monoterpenes, isoprene, and aromatics accounted for $48 \%$, 
$35 \%$, and $17 \%$ of their simulated SOC, respectively. All of the modeling studies described above considered only SOC produced by equilibrium-partitioning of semi-volatile oxidation products of VOC precursors (Pankow et al., 1994a, b; Odum et al., 1996). Recent studies indicate that additional secondary formation pathways, such as the aqueous reactions of dicarbonyls and the condensation of semi-volatile and intermediate volatility organic compounds, may be important (Robinson et al., 2007; Carlton et al., 2009; Hallquist et al., 2009; Jimenez et al., 2009).

A major obstacle in understanding the sources and distributions of Chinese carbonaceous aerosol is the lack of a consistent measurement network in China with good spatiotemporal coverage, such as the IMPROVE network in the United States (Malm et al., 1994). In the past decade, many studies have measured EC and OC at Chinese urban and rural sites. However, most of such measurements were conducted at a single location for a short period of time. Only two recent published studies measured carbonaceous aerosols simultaneously at multiple locations across China in winter and summer (Cao et al., 2007) or over an entire year (X. Y. Zhang et al., 2008). The spatial and seasonal variability of these measurements provide important information about the sources of carbonaceous aerosols.

The goal of this study was to quantify the primary and secondary sources of Chinese carbonaceous aerosols by comparing numerical simulations against observations countrywide for all seasons. A database of surface EC and OC measurements at background, rural, and urban locations was compiled from previous studies to represent the spatial and seasonal variability. We conducted a year-long simulation of carbonaceous aerosols in China, incorporating the current knowledge of primary sources and secondary formation pathways. The simulated results were then compared to seasonal surface observations at background and rural sites in China to derive "top-down" estimates for the primary EC and OC sources and to quantify the secondary formation of OC.

\section{Model and data description}

\subsection{GEOS-Chem model}

We simulated carbonaceous aerosol over East and South Asia using the one-way nested-grid capability of the GEOSChem 3-D chemical transport model (version 8.1.3; http: //acmg.seas.harvard.edu/geos/). GEOS-Chem is driven by assimilated meteorological data from the Goddard Earth Observing System (GEOS-5) of the NASA Global Modeling Assimilation Office (Bey et al., 2001). Meteorology fields in GEOS-5 have a temporal resolution of $6 \mathrm{~h}(3 \mathrm{~h}$ for surface variables), a horizontal resolution of $0.667^{\circ}$ longitude $\times 0.5^{\circ}$ latitude, and 72 hybrid eta levels in the vertical direction extending from the surface to $0.01 \mathrm{hPa}$. To drive GEOS-Chem, the number of vertical levels was reduced to 47 by merging layers in the stratosphere. The lowest $2 \mathrm{~km}$ of the atmosphere was resolved by 14 levels. For global simulations, the horizontal resolution of meteorology fields was reduced to either $5^{\circ}$ longitude $\times 4^{\circ}$ latitude or $2.5^{\circ}$ longitude $\times 2^{\circ}$ latitude.

The one-way nested-grid capability of GEOS-Chem for East and South Asia $\left(70-150^{\circ} \mathrm{E}, 10^{\circ} \mathrm{S}-55^{\circ} \mathrm{N}\right)$ was developed by Wang et al. (2004) and updated by Chen et al. (2009) for GEOS-5 using the native horizontal resolution $\left(0.667^{\circ}\right.$ longitude $\times 0.5^{\circ}$ latitude). Tracer concentrations at the lateral boundaries were provided by a global GEOS-Chem simulation at $5^{\circ}$ longitude $\times 4^{\circ}$ latitude horizontal resolution and updated in the nested-grid model every $3 \mathrm{~h}$. Chen et al. (2009) simulated the summertime distribution and transport of CO in China. They showed that the nested-grid model is able to resolve individual cities associated with high emission intensities, as well as reproduce the transport of urban plumes to a suburban site near Beijing. Y. Wang et al. (2009) compared results from the nested-grid model against $\mathrm{O}_{3}, \mathrm{CO}$, $\mathrm{NO}_{\mathrm{y}}$, and $\mathrm{SO}_{2}$ measurements at a rural site downwind of Beijing. They found that the model is able to reproduce the observed pollution level changes associated with emission reductions in Beijing.

Carbonaceous aerosols in the standard GEOS-Chem model include primary EC, primary OC, as well as SOC as part of SOA produced from VOC precursor oxidation. The terms "elemental carbon aerosol" (EC) and "black carbon aerosol" (BC) are often used interchangeably in emission and modeling literature, and there are currently no universally accepted definitions for the two terms. From a measurement stand point, EC mostly refers to aerosol measured with thermal/optical techniques, while BC mostly refers to aerosol measured with photo-absorption techniques. The mass concentrations determined using these two techniques can sometimes be significantly different (Jeong et al., 2004). For the purpose of this study, we considered $\mathrm{EC}$ and $\mathrm{BC}$ to be equivalent. The "bottom-up" emission inventories of EC and OC and VOC precursors used to drive our model are described in Sect. 2.2. EC and OC are separated into hydrophilic and hydrophobic components and are removed by dry deposition (Wesley, 1989; Wang et al., 1998) and wet deposition (Liu et al., 2001; Mari et al., 2000). Freshly emitted EC and OC are assumed to be $20 \%$ and $50 \%$ water-soluble, respectively (Cooke et al., 1999; Park et al., 2005). The water-insoluble fraction is converted to water-soluble in the atmosphere with an e-folding lifetime of 1.15 days, constrained by observed export efficiency vertical profiles (Park et al., 2005). We conducted a sensitivity test by tripling this conversion lifetime in the nested-grid model but found the change in simulated monthly mean Chinese surface EC and OC concentrations to be less than $5 \%$.

GEOS-Chem simulates SOA formation by equilibrium partitioning of semi-volatile oxidation products from biogenic terpenes, (Liao et al., 2007), biogenic isoprene (Henze and Seinfeld, 2006), as well as aromatics from anthropogenic activities and biomass burning (Henze et al., 2008). The 
equilibrium partitioning was as described by Chung and Seinfeld (2002), except that in this study the semi-volatile SOA was allowed to partition onto all preexisting OC and inorganic aqueous aerosols. This provided an upper estimate of semi-volatile SOA production. We assumed that all semivolatile SOA have an aerosol-to-carbon (SOA/SOC) mass ratio of 2.1 (Turpin and Lim, 2001) and are $80 \%$ water-soluble following Chung and Seinfeld (2002).

GEOS-Chem also simulates SOA produced from the irreversible uptake of glyoxal and methylglyoxal by aqueous particles as described in Fu et al. (2008, 2009). Glyoxal and methylglyoxal are two dicarbonyls produced during the oxidation of many biogenic and anthropogenic VOCs, including most importantly isoprene, with minor contributions from acetone, acetylene, alkenes, and aromatics (Fu et al., 2008; Myriokefalitakis et al., 2008). Recent laboratory studies and in situ measurements indicate uptake of glyoxal and methylglyoxal by aqueous particles to form SOA (Schweitzer et al., 1998; Liggio et al., 2005a, b; Volkamer et al., 2007, 2009; Galloway et al., 2009; Sareen et al., 2010; De Haan et al., 2011). We represented this process as a first-order reactive uptake by aqueous aerosols and cloud droplets with a reactive uptake coefficient of $\gamma=2.9 \times 10^{-3}$, following the chamber study by Liggio et al. (2005) for glyoxal. The same uptake coefficient was adopted for methylglyoxal, consistent with the laboratory results by Zhao et al. (2006). Although uncertainty remains regarding the mechanism of SOA formation by dicarbonyls (Kroll et al., 2005), our model representation provides a state-of-the-science estimate of this secondary OC source. Dicarbonyl SOA is assumed to be fully water-soluble. Additional SOA production from semivolatile and intermediate volatility organic compounds have recently been developed for GEOS-Chem (Pye and Seinfeld, 2010), but that pathway was not included in the present study.

Our simulation was conducted from July 2005 to December 2006; the first six months initialized the model. Results from January to December 2006 were analyzed. We also conducted sensitivity tests by turning off Chinese EC and OC emissions from each of the three source sectors (anthropogenic non-residential, anthropogenic residential, and biomass burning), in turn and all at once. This was done to evaluate the contributions to surface concentrations from each source sector and from non-Chinese sources.

\subsection{Bottom-up emission inventories of EC, OC, and VOC precursors}

As a starting point for our analysis, we used state-of-thescience bottom-up emission inventories for EC, OC, and VOC precursors over China and the rest of East and South Asia to drive our model simulation. Table 1 summarizes the bottom-up emission estimates for Chinese carbonaceous aerosols. Table 2 summarizes the bottom-up Chinese emissions of VOC that are precursors to SOC in our model.
Anthropogenic emissions of EC and OC for China and the rest of East and South Asia were taken from Zhang et al. (2009), developed for the year 2006, including nonresidential and residential sources. Non-residential sources included power generation, industry, and transportation. Residential sources included combustion of fossil fuel and biofuel, as well as non-combustive activities. The inventory was based on national and provincial (for China) statistics for the year 2006 or extrapolated statistics from the years 2004 and 2005 , then spatially allocated to $0.5^{\circ}$ longitude $\times 0.5^{\circ}$ latitude resolution using spatial surrogates. Emission factors were developed using a combination of Chinese measurements, estimates, and data from western sources (Zhang et al., 2007). The estimated anthropogenic non-residential emissions of $\mathrm{EC}$ and $\mathrm{OC}$ were $0.81 \mathrm{TgC} \mathrm{yr}^{-1}$ and $0.61 \mathrm{TgC} \mathrm{yr}^{-1}$, respectively, with industry being the largest non-residential source for both EC and OC (mostly from coke and brick production), followed by transportation and power generation. The estimated Chinese residential emissions of $\mathrm{EC}$ and $\mathrm{OC}$ were $1.00 \mathrm{TgC} \mathrm{yr}^{-1}$ and $2.61 \mathrm{TgC} \mathrm{yr}^{-1}$, respectively. Total anthropogenic EC emission was $1.81 \mathrm{TgC} \mathrm{yr}^{-1}$ with $\pm 208 \%$ uncertainty (95\% confidence interval). Total anthropogenic OC emission was $3.22 \mathrm{TgC} \mathrm{yr}^{-1}$ with $\pm 258 \%$ uncertainty. Seasonal variations of anthropogenic emissions in China were calculated by assuming a dependence of stove operation on regional monthly mean temperatures for the residential sector, and by using provincial level monthly activity data for the non-residential sector (Zhang et al., 2009). We assumed no seasonal variation of anthropogenic emissions outside of China.

Biomass burning emissions of $\mathrm{EC}$ and $\mathrm{OC}$ for China and rest of East and South Asia were taken from Streets et al. (2003b), which represents average burning activities for the mid-1990s. Several biomass burning emission inventories have been developed more recently based on satellite burnt area observations (van der Werf et al., 2006, 2010; Song et al., 2010). However, these inventories either underestimate or entirely exclude the contribution from in-field crop residue burning, which has been shown to be an important seasonal biomass burning source of carbonaceous aerosols in China (Yang et al., 2008; T. Zhang et al., 2008; Huang et al., 2012). We used the inventory developed by Streets et al. (2003b) for the sake of completeness in the present study. A very recent study by Huang et al. (2012) provided new emission estimates for the in-field crop residue burning in China, and we will explore the use of that inventory in a future study. However, as will be shown in Sect. 4.1, the measurements that we used to constrain EC sources are not sensitive to biomass burning emissions. Thus, the choice of biomass burning emission inventories did not have a large impact on the top-down estimates of anthropogenic EC emissions presented in Sect. 4.1.

We compounded the annual biomass burning fluxes from Streets et al. (2003b) with the monthly variation from Duncan et al. (2003), which was based on the Along Track 
Table 1. Chinese carbonaceous aerosol emission estimates.

\begin{tabular}{l|ll|lll}
\hline \multirow{2}{*}{ Source sectors } & \multicolumn{2}{|c|}{ EC [TgC yr $\left.{ }^{-1}\right]$} & \multicolumn{2}{c}{ OC [TgC $\left.\mathrm{yr}^{-1}\right]$} \\
\cline { 2 - 6 } & Bottom-up & Top-down & Bottom-up & Hybrid $^{\mathrm{e}}$ & Top-down \\
\hline $\begin{array}{c}\text { Anthropogenic } \\
\text { non-residential }\end{array}$ & $0.81^{\mathrm{a}}$ & $2.94 \pm 0.60( \pm 40 \%)^{\mathrm{c}}$ & & 3.41 & $6.42 \pm 1.10( \pm 34 \%)^{\mathrm{c}}$ \\
$\begin{array}{c}\text { residential } \\
\text { Biomass burning }\end{array}$ & $1.00^{\mathrm{a}}$ & & $0.61^{\mathrm{a}}$ & & \\
\hline China total & 1.92 & $3.05 \pm 0.78\left( \pm 50 \% 1^{\mathrm{b}}\right.$ & 3.95 & 4.14 & $6.67 \pm 1.30( \pm 38 \%)^{\mathrm{c}}$ \\
\hline
\end{tabular}

a From Zhang et al. (2009). Uncertainties, represented as $95 \%$ confidence intervals (CI), are $\pm 208 \%$ for EC and $\pm 258 \%$ for OC.

${ }^{\mathrm{b}}$ From Streets et al. (2003b). Uncertainties, represented as $95 \% \mathrm{CI}$, are $\pm 450 \%$ for EC and $\pm 420 \%$ for OC.

c Uncertainties are represented as \pm standard deviations. The corresponding $95 \% \mathrm{CI}$ are calculated assuming normal distribution and are shown in parentheses. See text for details

${ }^{\mathrm{d}}$ Bottom-up emissions and uncertainties are adopted since observational constraints are not available.

${ }^{\mathrm{e}}$ Uncertainties are not calculated but are expected to be similar to those of the bottom-up emissions.

Table 2. Volatile organic compound (VOC) precursors of secondary organic carbon aerosols (SOC) in China.

\begin{tabular}{|c|c|c|c|c|c|c|c|}
\hline \multirow[t]{2}{*}{ VOC precursor } & \multicolumn{4}{|c|}{ Chinese emission $^{\mathrm{a}}\left[\mathrm{Tg} \mathrm{yr}^{-1}\right]$} & \multicolumn{3}{|c|}{$\begin{array}{l}\text { Simulated contribution to annual mean surface } \\
\text { OC concentration in } \mathrm{China}^{\mathrm{b}}\left[\mu \mathrm{gC} \mathrm{m}{ }^{-3}\right]\end{array}$} \\
\hline & Anthropogenic ${ }^{c}$ & Biomass burning & Biogenic & Total & Semi-volatile SOC & Dicarbonyl SOC ${ }^{\mathrm{d}}$ & Total SOC \\
\hline Isoprene & - & - & 10.5 & 10.5 & 0.10 & 0.29 & 0.39 \\
\hline Monoterpenes & - & - & 5.6 & 5.6 & 0.21 & 0.01 & 0.23 \\
\hline Aromatics ${ }^{\mathrm{e}}$ & 5.3 & 0.11 & - & 5.4 & 0.09 & 0.08 & 0.17 \\
\hline Ethylene & 2.2 & 1.6 & 0.2 & 4.1 & - & 0.04 & 0.04 \\
\hline Sesquiterpenes & - & - & 0.6 & 0.6 & 0.04 & - & 0.04 \\
\hline Alcohols & - & - & 1.6 & 1.6 & 0.03 & - & 0.03 \\
\hline$\geq \mathrm{C}_{4}$ alkanes & 4.8 & 0.1 & - & 4.8 & - & 0.02 & 0.02 \\
\hline Acetylene & 2.4 & 0.1 & - & 2.5 & - & 0.02 & 0.02 \\
\hline Acetone & 0.2 & 0.3 & 1.6 & 2.1 & - & 0.02 & 0.02 \\
\hline$\geq \mathrm{C}_{3}$ alkenes & 0.6 & 1.7 & 0.3 & 2.6 & - & 0.01 & 0.01 \\
\hline Glyoxal & - & 0.2 & - & 0.2 & - & 0.01 & 0.01 \\
\hline Methylglyoxal & - & 0.1 & - & 0.1 & - & 0.01 & 0.01 \\
\hline Hydroxyacetone & - & 0.1 & - & 0.1 & - & 0.006 & 0.006 \\
\hline Propane & 0.4 & 0.1 & - & 0.5 & - & 0.005 & 0.005 \\
\hline Glycolaldehyde & - & 0.2 & - & 0.2 & - & 0.001 & 0.001 \\
\hline Total & 15.9 & 4.6 & 20.4 & 40.9 & 0.48 & 0.53 & 1.01 \\
\hline
\end{tabular}

a Represents primary emissions only.

b From GEOS-Chem simulation using the top-down EC and OC emission estimates described in the text.

${ }^{c}$ Includes anthropogenic residential and non-residential sources.

${ }^{\mathrm{d}}$ Relative contributions to dicarbonyl SOC from individual VOC precursors are estimated using dicarbonyl molar yields calculated by Fu et al. (2007) based on a one-year GEOSChem global simulation.

e Includes benzene, toluene, and xylenes.

Scanning Radiometer (ATSR) satellite hot spot counts from 1996 to 2000. Total Chinese biomass burning emission of

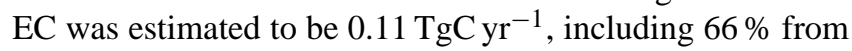
crop residue, $22 \%$ from grassland, and $12 \%$ from forest. Total Chinese biomass burning emission estimate for OC was $0.71 \mathrm{TgC} \mathrm{yr}^{-1}$, including $50 \%$ from crop residue, $26 \%$ from forest, and $24 \%$ from grassland. The uncertainties for these estimates were $\pm 450 \%$ (EC) and $\pm 420 \%$ (OC) (Streets et al., 2003b).
Figures 1 and 2 show the spatial distributions of annual $\mathrm{EC}$ and $\mathrm{OC}$ emissions in the bottom-up inventories. The spatial distributions for EC and OC emissions are very similar in each sector, reflecting their common combustive origin. Non-residential anthropogenic emissions are highest over the heavily industrialized areas of northern China, as well as over the Yangtze River Delta (YRD) and Pearl River Delta (PRD) megacity clusters. Residential emissions are high over densely populated areas, including northern China, Sichuan 

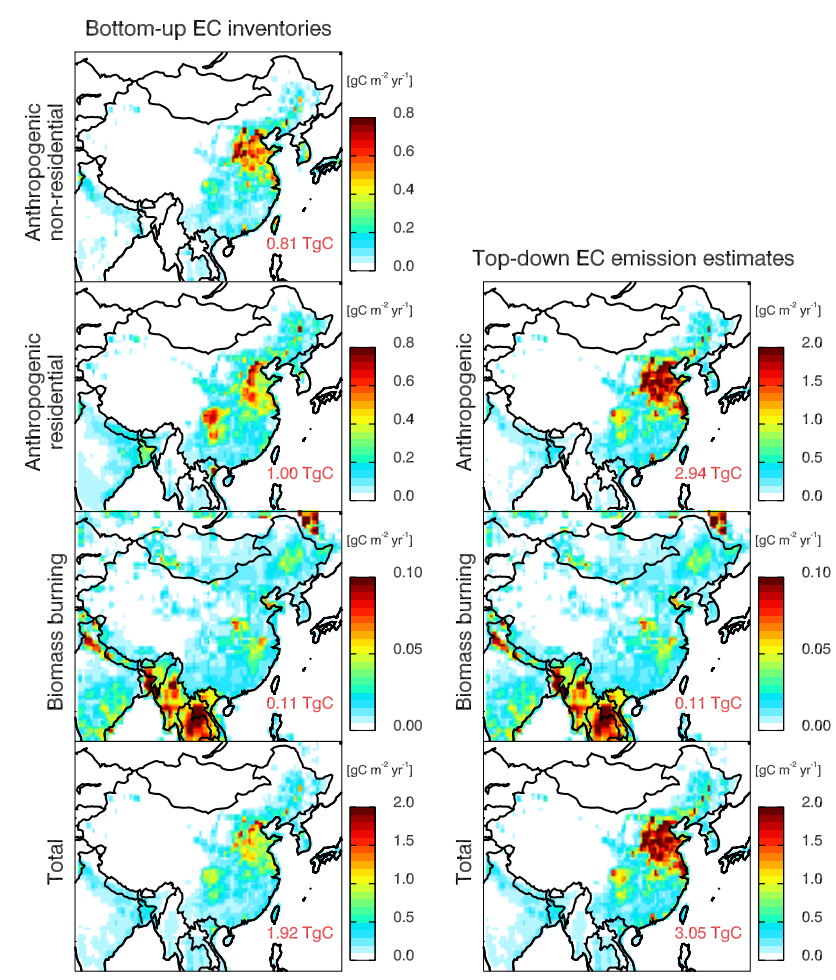

Fig. 1. Annual emissions of EC from China. Left panel: bottomup inventories of Zhang et al. (2009) (anthropogenic) and Streets et al. (2003b) (biomass burning). Right panel: emissions inferred from surface EC obserations on the basis of the multiple regression analysis in this study. Biomass burning emission is unchanged because no observational constrain is available. Chinese annual emission totals are shown inset in red.

Basin, Northeastern China Plain, and the YRD and PRD megacity clusters, largely overlapping with industrialized areas. Within China, biomass burning emissions are highest over central and northeastern China from crop residue burning. Outside China, biomass burning emissions are high over the Indochina Peninsula due to land clearing before the local growing season (spring), which can affect the air quality in southern China through transport (Deng et al., 2008).

Table 2 summarizes the emissions of VOC species that are SOC precursors in our model. Biogenic isoprene emissions were calculated with the MEGAN algorithm (Guenther et al., 2006), then scaled to match satellite formaldehyde observations (Fu et al., 2007) to an annual emission of $10.5 \mathrm{Tg} \mathrm{yr}^{-1}$. Biogenic monoterpenes, alcohol, and sesquiterpene emissions were based on the GEIA inventory (Guenther et al., 1995). Other biogenic VOC precursors included acetone, emissions of which followed Jacob et al. (2002), as well as ethylene and higher alkene emissions, which were scaled to isoprene using emission ratios from Goldstein et al. (1996). Anthropogenic VOC emissions were from the Zhang et al. (2009) inventory described above, with the VOC species mapped to match the GEOS-
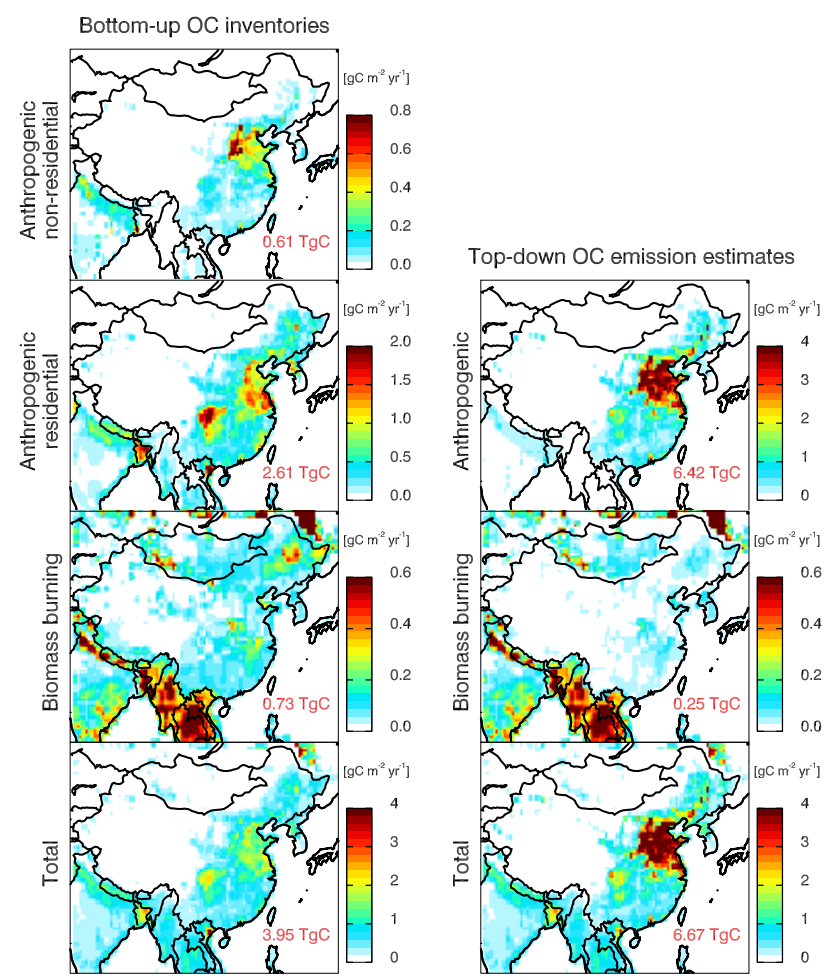

Fig. 2. Annual emissions of OC from China. Left panel: bottomup inventories of Zhang et al. (2009) (anthropogenic) and Streets et al. (2003b) (biomass burning). Right panel: OC emissions inferred by scaling top-down EC emission estimates with observed primary OC/EC ratios by Cao et al. (2007). See text for details. Chinese annual emission totals are shown inset in red.

Chem chemical mechanism. Biomass burning VOC emissions were from Streets et al. (2003b), with alkene, xylenes, and formaldehyde emissions scaled to match satellite constraints (Fu et al., 2007). Biomass burning glyoxal, methylglyoxal, glycolaldehyde, and hydroxyacetone were scaled to biomass burning CO emissions (Streets et al., 2003b) following Fu et al. (2008).

Figure 3 shows the spatial distributions of isoprene, monoterpenes, and toluene emissions. Biogenic VOC are mostly emitted in eastern China, reflecting the distribution of vegetation and precipitation. Highest emissions are found over the forested areas in northeastern, central, and southeastern China. Toluene emissions (mostly anthropogenic) are highest over the heavily populated and industrialized North China Plain and Sichuan Basin, as well as over the coastal megacities.

\subsection{Carbonaceous aerosol measurements}

To constrain the annual sources of carbonaceous aerosols at the country-wide level, we needed measurements with sufficient coverage in time and space to allow assessment of seasonal variation and spatial distribution. At the time of 
(a) Isoprene

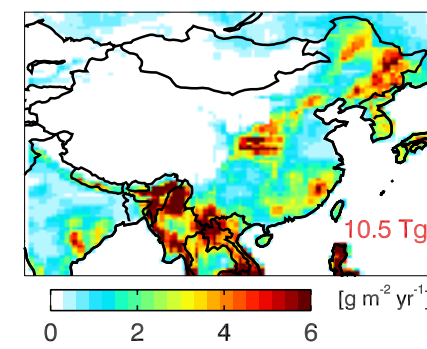

(b) Monoterpenes

(c) Toluenes

Fig. 3. Annual emissions of (a) biogenic isoprene, (b) biogenic monoterpene, and (c) anthropogenic and biomass burning toluene in the model. Chinese annual emission totals are shown inset in red.

this study, there was one carbonaceous aerosol measurement network in China (X. Y. Zhang et al., 2008), but only one year's worth of data was published and there were gaps in the spatial coverage. Therefore, we compiled a database of monthly mean EC and OC surface measurements from previous studies. Our data selection criteria were that the measurements (1) provided information on seasonal variation, (2) combined to cover different parts of China, (3) were analyzed in a consistent manner, and (4) preferably were conducted at background or rural locations to represent regional pollutant levels.

Based on the above criteria, we selected measurements from five studies conducted between the years 2003 and 2006, including three background sites, seven rural sites, and twenty-one urban sites (X. Y. Zhang et al., 2008; Qu et al., 2008; Cao et al., 2007, 2009; Y. M. Han et al., 2008). Table 3 lists the details of the measurements. Figure 4 shows the spatial distribution of the measurement sites. Two of these studies (X. Y. Zhang et al., 2008; Qu et al., 2008) sampled $\mathrm{PM}_{10}$ and analyzed EC and OC contents in the same laboratory at the Chinese Academy of Meteorological Sciences. The other three studies sampled $\mathrm{PM}_{2.5}$ (Cao et al., 2007; Y. M. Han et al., 2008) or total suspended particles (TSP) (Cao et al., 2009) and analyzed the EC and OC contents at the Institute of Earth Environment, Chinese Academy of Sciences. All analyses followed the IMPROVE (Interagency Monitoring of Protected Visual Environments) thermal/optical reflectance (TOR) protocol (Chow et al., 1993, 2004) using DRI model 2001 Carbon Analyzer. Thus, there was some level of consistency and comparability across the measurements. Only EC concentrations at background and rural sites (all sampled as $\mathrm{PM}_{10}$ or TSP) were used in the multiple regression to constrain emissions as described in Sect. 4. Both EC and OC concentrations had considerable day-to-day variability at all sites. The normalized standard deviations of the monthly mean concentrations at background and rural sites were $46 \%$ for EC and $33 \%$ for OC.

Table 3 summarizes the annual mean surface EC and OC concentrations measured at the 31 background, rural and urban sites. Urban annual mean surface concentrations

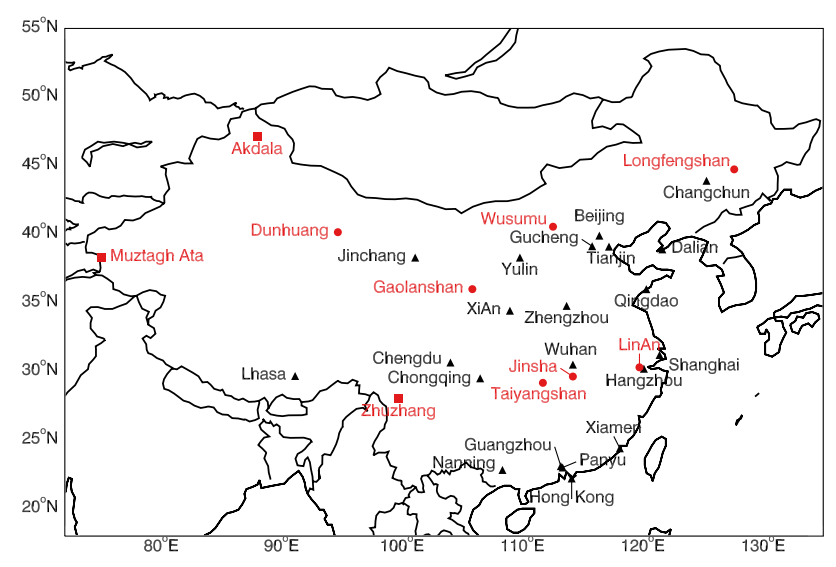

Fig. 4. Locations of the Chinese surface measurement sites used in this study, including three background sites (red squares), seven rural sites (red circles), and twenty-one urban sites (black triangles).

ranged from $2.7-14 \mu \mathrm{gC} \mathrm{m}{ }^{-3}$ for $\mathrm{EC}$ and $8.2-52 \mu \mathrm{gC} \mathrm{m}{ }^{-3}$ for OC. Rural annual mean surface concentrations ranged from $2.4-4.7 \mu \mathrm{gC} \mathrm{m}^{-3}$ for EC and $12-40 \mu \mathrm{gC} \mathrm{m}^{-3}$ for OC. These values are significantly higher than the range of annual mean concentrations measured in urban $\left(0.9-1.8 \mu \mathrm{gC} \mathrm{m}^{-3}\right.$ EC, $\left.2.0-5.9 \mu \mathrm{gC} \mathrm{m}^{-3} \mathrm{OC}\right)$ and rural $\left(0.02-1.8 \mu \mathrm{gC} \mathrm{m}{ }^{-3} \mathrm{EC}\right.$, $0.07-7.8 \mu \mathrm{gC} \mathrm{m}{ }^{-3} \mathrm{OC}$ ) air in North America and in Europe (Hand et al., 2011; Yttri et al., 2007).

\section{Model evaluation}

We compared simulated surface carbonaceous aerosol concentrations against observations to evaluate our model. In general, model results cannot be evaluated on a 24 -h-average basis in continental surface air, because nighttime stratification is often poorly resolved (Jacob et al., 1993). This was not an issue in our case because our model had high vertical resolution near the surface. The situation that our model could not resolve was where the nighttime Obukhov length is positive and shallower than the bottom model layer (in our case approximately $100 \mathrm{~m}$ ). We conducted a sensitivity test 
Table 3. List of Chinese surface EC and OC concentration observations used in this study.

\begin{tabular}{|c|c|c|c|c|c|c|c|c|}
\hline \multirow[b]{2}{*}{ Site $^{\mathrm{a}}$} & \multirow[b]{2}{*}{$\begin{array}{l}\text { Aerosol } \\
\text { size } \\
\text { sampled }^{b}\end{array}$} & \multirow[b]{2}{*}{$\begin{array}{l}\text { Filter accumulation } \\
\text { period/sampling } \\
\text { frequency }\end{array}$} & \multirow[b]{2}{*}{$\begin{array}{l}\text { Observation } \\
\text { period }\end{array}$} & \multirow[b]{2}{*}{$\begin{array}{l}\text { Observed } \\
\text { annual mean } \\
\text { EC/OC surface } \\
\text { concentrations } \\
{\left[\mu \mathrm{gC} \mathrm{m}^{-3}\right]}\end{array}$} & \multicolumn{2}{|c|}{$\begin{array}{l}\text { Simulated annual mean EC/OC surface } \\
\text { concentrations }{ }^{d}\left[\mu \mathrm{gC} \mathrm{m}^{-3}\right]\end{array}$} & \multirow[b]{2}{*}{$\begin{array}{l}\text { Used in } \\
\text { multiple } \\
\text { regression to } \\
\text { constrain EC } \\
\text { emissions? }\end{array}$} & \multirow[b]{2}{*}{ Reference $^{\mathrm{e}}$} \\
\hline & & & & & $\begin{array}{l}\text { using "bottom- } \\
\text { up" emission } \\
\text { inventories }{ }^{\mathrm{d}}\end{array}$ & $\begin{array}{l}\text { using "top- } \\
\text { down" } \\
\text { emission } \\
\text { estimates }\end{array}$ & & \\
\hline \multicolumn{9}{|l|}{ Background } \\
\hline $\begin{array}{l}\text { Akdala } \\
\left(47.1^{\circ} \mathrm{N}\right. \\
\left.87.97^{\circ} \mathrm{E}\right)\end{array}$ & $\mathrm{PM}_{10}$ & 72 h/every 3-10 days & $\begin{array}{l}\text { Aug, Sep, Nov, } \\
\text { and Dec 2004; } \\
\text { Jan-Mar } 2005\end{array}$ & $0.33 / 2.8$ & $0.23 / 0.78$ & $0.23 / 0.83$ & Yes & {$[1]$} \\
\hline $\begin{array}{l}\text { Muztagh Ata } \\
\left(38.3^{\circ} \mathrm{N},\right. \\
\left.75.01^{\circ} \mathrm{E}\right)\end{array}$ & TSP & $\sim 7$ days ${ }^{\mathrm{f}} /$ weekly & $2005^{\mathrm{g}}$ & $0.051 / 0.51$ & $0.093 / 0.49$ & $0.10 / 0.47$ & Yes & [2] \\
\hline $\begin{array}{l}\text { Zhuzhang } \\
\left(28^{\circ} \mathrm{N},\right. \\
\left.99.72^{\circ} \mathrm{E}\right)\end{array}$ & $\mathrm{PM}_{10}$ & 72 h/every 3-4 days & $\begin{array}{l}\text { Aug-Dec 2004; } \\
\text { Jan-Feb } 2005\end{array}$ & $0.35 / 3.1$ & $0.15 / 1.1$ & $0.18 / 0.98$ & Yes & {$[1]$} \\
\hline \multicolumn{9}{|l|}{ Rural } \\
\hline $\begin{array}{l}\text { Dunhuang } \\
\left(40.15^{\circ} \mathrm{N} \text {, }\right. \\
\left.94.68^{\circ} \mathrm{E}\right)\end{array}$ & $\mathrm{PM}_{10}$ & 24 h/every 3 days & 2006 & $4.1 / 29$ & $0.19 / 0.88$ & $0.22 / 0.82$ & Yes & [3] \\
\hline $\begin{array}{l}\text { Gaolanshan } \\
\left(36^{\circ} \mathrm{N},\right. \\
\left.105.85^{\circ} \mathrm{E}\right)\end{array}$ & $\mathrm{PM}_{10}$ & 24 h/every 3 days & 2006 & $3.7 / 18$ & $1.0 / 2.7$ & $1.3 / 4.1$ & Yes & [3] \\
\hline $\begin{array}{l}\text { Wusumu } \\
\left(40.56^{\circ} \mathrm{N},\right. \\
\left.112.55^{\circ} \mathrm{E}\right)\end{array}$ & $\mathrm{PM}_{10}$ & 24 h/daily & $\begin{array}{l}\text { Sep 2005; Jan } \\
\text { and Jul 2006; } \\
\text { May } 2007\end{array}$ & $3.7 / 27$ & $1.8 / 4.3$ & $3.2 / 8.9$ & Yes & {$[4]$} \\
\hline $\begin{array}{l}\text { Longfengshan } \\
\left(44.73^{\circ} \mathrm{N} \text {, }\right. \\
\left.127.6^{\circ} \mathrm{E}\right)\end{array}$ & $\mathrm{PM}_{10}$ & 24 h/every 3 days & 2006 & $2.4 / 16$ & $1.6 / 4.7$ & $2.3 / 7.4$ & Yes & {$[3]$} \\
\hline $\begin{array}{l}\text { Taiyangshan } \\
\left(29.17^{\circ} \mathrm{N},\right. \\
\left.111.71^{\circ} \mathrm{E}\right)\end{array}$ & $\mathrm{PM}_{10}$ & 24 h/every 3 days & 2006 & $2.4 / 12$ & $2.5 / 7.5$ & $3.9 / 11$ & Yes & [3] \\
\hline $\begin{array}{l}\text { Jinsha } \\
\left(29.63^{\circ} \mathrm{N},\right. \\
\left.114.2^{\circ} \mathrm{E}\right)\end{array}$ & $\mathrm{PM}_{10}$ & 24 h/every 3 days & Jun-Nov 2006 & $3.0 / 14$ & $1.9 / 6.8$ & $3.5 / 10$ & Yes & [3] \\
\hline $\begin{array}{l}\text { LinAn } \\
\left(30.3^{\circ} \mathrm{N}\right. \\
\left.119.73^{\circ} \mathrm{E}\right)\end{array}$ & $\mathrm{PM}_{10}$ & 24 h/every 3 days & 2004-2005 & $4.8 / 15$ & $2.0 / 4.9$ & $4.1 / 9.4$ & Yes & [3] \\
\hline \multicolumn{9}{|l|}{ Urban } \\
\hline $\begin{array}{l}\text { Jinchang } \\
\left(38.3^{\circ} \mathrm{N}\right. \\
\left.101.1^{\circ} \mathrm{E}\right)\end{array}$ & $\mathrm{PM}_{2.5}$ & 24 h/daily & $\begin{array}{l}\text { Jan, Jul, and } \\
\text { Aug } 2003\end{array}$ & $3.4 / 16$ & $0.40 / 1.5$ & $0.43 / 1.6$ & No & {$[5]$} \\
\hline $\begin{array}{l}\text { Yulin }\left(38.3^{\circ} \mathrm{N}\right. \\
\left.109.8^{\circ} \mathrm{E}\right)\end{array}$ & $\mathrm{PM}_{2.5}$ & 24 h/daily & $\begin{array}{l}\text { Jan, Jul, and } \\
\text { Aug } 2003\end{array}$ & $6.2 / 24$ & $1.4 / 4.4$ & $1.8 / 6.2$ & No & [5] \\
\hline $\begin{array}{l}\text { Guchengh } \\
\left(39.13^{\circ} \mathrm{N}\right. \\
\left.115.8^{\circ} \mathrm{E}\right)\end{array}$ & $\mathrm{PM}_{10}$ & 24 h/every 3 days & 2006 & $12 / 40$ & $4.9 / 9.0$ & $8.1 / 22$ & No & {$[3]$} \\
\hline $\begin{array}{l}\text { Beijing } \\
\left(39.9^{\circ} \mathrm{N}\right. \\
\left.116.4^{\circ} \mathrm{E}\right)\end{array}$ & $\mathrm{PM}_{2.5}$ & 24 h/daily & $\begin{array}{l}\text { Jan and Jul } \\
2003\end{array}$ & $6.2 / 23$ & $6.9 / 13$ & $9.3 / 27$ & No & {$[5]$} \\
\hline $\begin{array}{l}\text { Tianjin } \\
\left(39.1^{\circ} \mathrm{N},\right. \\
\left.117.2^{\circ} \mathrm{E}\right)\end{array}$ & $\mathrm{PM}_{2.5}$ & 24 h/daily & $\begin{array}{l}\text { Jan and Jul } \\
2003\end{array}$ & $6.1 / 28$ & $4.5 / 8.9$ & $7.0 / 20$ & No & [5] \\
\hline $\begin{array}{l}\text { Dalian } \\
\left(38.9^{\circ} \mathrm{N}\right. \\
\left.121.63^{\circ} \mathrm{E}\right)\end{array}$ & $\mathrm{PM}_{10}$ & 24 h/every 3 days & 2006 & $5.4 / 18$ & $1.5 / 4.1$ & $2.4 / 7.2$ & No & [3] \\
\hline $\begin{array}{l}\text { Changchun } \\
\left(43.9^{\circ} \mathrm{N}\right. \\
\left.125.3^{\circ} \mathrm{E}\right)\end{array}$ & $\mathrm{PM}_{2.5}$ & 24 h/daily & $\begin{array}{l}\text { Jan, Jul, and } \\
\text { Aug } 2003\end{array}$ & $8.2 / 26$ & $3.2 / 7.0$ & $4.7 / 14$ & No & {$[5]$} \\
\hline
\end{tabular}


Table 3. Continued.

\begin{tabular}{|c|c|c|c|c|c|c|c|c|}
\hline \multirow[b]{2}{*}{ Site $^{\mathrm{a}}$} & \multirow[b]{2}{*}{$\begin{array}{l}\text { Aerosol } \\
\text { size } \\
\text { sampled }^{b}\end{array}$} & \multirow[b]{2}{*}{$\begin{array}{l}\text { Filter accumulation } \\
\text { period/sampling } \\
\text { frequency }\end{array}$} & \multirow[b]{2}{*}{$\begin{array}{l}\text { Observation } \\
\text { period }\end{array}$} & \multirow[b]{2}{*}{$\begin{array}{l}\text { Observed } \\
\text { annual mean } \\
\text { EC/OC surface } \\
\text { concentrations } \\
{\left[\mu \mathrm{gC} \mathrm{m}^{-3}\right]}\end{array}$} & \multicolumn{2}{|c|}{$\begin{array}{l}\text { Simulated annual mean EC/OC surface } \\
\text { concentrations }{ }^{d}\left[\mu \mathrm{gC} \mathrm{m}^{-3}\right]\end{array}$} & \multirow[b]{2}{*}{$\begin{array}{l}\text { Used in } \\
\text { multiple } \\
\text { regression to } \\
\text { constrain EC } \\
\text { emissions? }\end{array}$} & \multirow[b]{2}{*}{ Reference $^{\mathrm{e}}$} \\
\hline & & & & & $\begin{array}{l}\text { using "bottom- } \\
\text { up" emission } \\
\text { inventories }^{\mathrm{d}}\end{array}$ & $\begin{array}{l}\text { using "top- } \\
\text { down" } \\
\text { emission } \\
\text { estimates }\end{array}$ & & \\
\hline $\begin{array}{l}\mathrm{XiAn} \\
\left(34.43^{\circ} \mathrm{N},\right. \\
\left.108.97^{\circ} \mathrm{E}\right)\end{array}$ & $\mathrm{PM}_{10}$ & 24 h/every 3 days & $\begin{array}{l}\text { Jan-Aug 2006; } \\
\text { Oct-Dec } 2006\end{array}$ & $14 / 42$ & $2.9 / 6.1$ & $4.7 / 14$ & No & [3] \\
\hline $\begin{array}{l}\text { Zhengzhou } \\
\left(34.78^{\circ} \mathrm{N} \text {, }\right. \\
\left.113.68^{\circ} \mathrm{E}\right)\end{array}$ & $\mathrm{PM}_{10}$ & 24 h/every 3 days & Jan-Aug 2006 & $9.5 / 25$ & $4.7 / 10$ & $7.8 / 21$ & No & [3] \\
\hline $\begin{array}{l}\text { Qingdao } \\
\left(36^{\circ} \mathrm{N},\right. \\
\left.120.3^{\circ} \mathrm{E}\right)\end{array}$ & $\mathrm{PM}_{2.5}$ & 24 h/daily & $\begin{array}{l}\text { Jan and Jul } \\
2003\end{array}$ & $3.9 / 16$ & $1.9 / 4.7$ & $3.0 / 8.5$ & No & [5] \\
\hline $\begin{array}{l}\text { Lhasa } \\
\left(29.67^{\circ} \mathrm{N}\right. \\
\left.91.13^{\circ} \mathrm{E}\right)\end{array}$ & $\mathrm{PM}_{10}$ & 24 h/every 3 days & 2006 & $3.7 / 21$ & $0.20 / 1.1$ & $0.20 / 0.97$ & No & [3] \\
\hline $\begin{array}{l}\text { Chengdu } \\
\left(30.65^{\circ} \mathrm{N},\right. \\
\left.104.03^{\circ} \mathrm{E}\right)\end{array}$ & $\mathrm{PM}_{10}$ & 24 h/every 3 days & 2006 & $11 / 33$ & $2.7 / 7.0$ & $3.8 / 8.5$ & No & [3] \\
\hline $\begin{array}{l}\text { Chongqing } \\
\left(29.5^{\circ} \mathrm{N}\right. \\
\left.106.5^{\circ} \mathrm{E}\right)\end{array}$ & $\mathrm{PM}_{2.5}$ & $24 \mathrm{~h} /$ daily & $\begin{array}{l}\text { Jan and Jul } \\
2003\end{array}$ & $12 / 52$ & $4.7 / 13$ & $4.9 / 11$ & No & {$[5]$} \\
\hline $\begin{array}{l}\text { Wuhan } \\
\left(30.5^{\circ} \mathrm{N},\right. \\
\left.114.2^{\circ} \mathrm{E}\right)\end{array}$ & $\mathrm{PM}_{2.5}$ & 24 h/daily & $\begin{array}{l}\text { Jan and Jul } \\
2003\end{array}$ & $5.6 / 26$ & $4.0 / 10$ & $5.4 / 13$ & No & [5] \\
\hline $\begin{array}{l}\text { Hangzhou } \\
\left(30.2^{\circ} \mathrm{N}\right. \\
\left.120.1^{\circ} \mathrm{E}\right)\end{array}$ & $\mathrm{PM}_{2.5}$ & 24 h/daily & $\begin{array}{l}\text { Jan, Jul, and } \\
\text { Aug } 2003\end{array}$ & $6.5 / 24$ & $1.7 / 4.6$ & $3.2 / 7.6$ & No & [5] \\
\hline $\begin{array}{l}\text { Shanghai } \\
\left(31.2^{\circ} \mathrm{N} \text {, }\right. \\
\left.121.4^{\circ} \mathrm{E}\right)\end{array}$ & $\mathrm{PM}_{2.5}$ & 24 h/daily & $\begin{array}{l}\text { Jan and Jul } \\
2003\end{array}$ & $5.6 / 21$ & $1.8 / 2.9$ & $3.5 / 6.8$ & No & [5] \\
\hline $\begin{array}{l}\text { Nanning } \\
\left(22.82^{\circ} \mathrm{N},\right. \\
\left.108.35^{\circ} \mathrm{E}\right)\end{array}$ & $\mathrm{PM}_{10}$ & 24 h/every 3 days & 2006 & $3.7 / 15$ & $1.5 / 5.8$ & $2.4 / 7.0$ & No & [3] \\
\hline $\begin{array}{l}\text { Guangzhou } \\
\left(23.1^{\circ} \mathrm{N}\right. \\
\left.113.2^{\circ} \mathrm{E}\right)\end{array}$ & $\mathrm{PM}_{2.5}$ & 24 h/daily & $\begin{array}{l}\text { Jan and Jul } \\
2003\end{array}$ & $8.9 / 26$ & $2.4 / 7.0$ & $4.3 / 9.2$ & No & {$[5]$} \\
\hline $\begin{array}{l}\text { Panyu }\left(23^{\circ} \mathrm{N}\right. \\
\left.113.35^{\circ} \mathrm{E}\right)\end{array}$ & $\mathrm{PM}_{10}$ & 24 h/every 3 days & 2006 & $8.9 / 21$ & $2.5 / 7.3$ & $4.6 / 11$ & No & [3] \\
\hline $\begin{array}{l}\text { Hong Kong } \\
\left(22.2^{\circ} \mathrm{N},\right. \\
\left.114.1^{\circ} \mathrm{E}\right)\end{array}$ & $\mathrm{PM}_{2.5}$ & 24 h/daily & $\begin{array}{l}\text { Jan, Jul, and } \\
\text { Aug } 2003\end{array}$ & $4.7 / 9.3$ & $1.1 / 3.6$ & $1.8 / 4.8$ & No & [5] \\
\hline $\begin{array}{l}\text { Xiamen } \\
\left(24.4^{\circ} \mathrm{N},\right. \\
\left.118.1^{\circ} \mathrm{E}\right)\end{array}$ & $\mathrm{PM}_{2.5}$ & 24 h/daily & $\begin{array}{l}\text { Jan, Jul, and } \\
\text { Aug } 2003\end{array}$ & $3.3 / 11$ & $1.3 / 4.3$ & $1.7 / 4.1$ & No & [5] \\
\hline
\end{tabular}

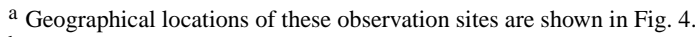

b Aerosol size sampled: particulate matter with aerodynamic diameter $<10 \mu \mathrm{m}\left(\mathrm{PM}_{10}\right)$, particulate matter with aerodynamic diameter $<2.5 \mu \mathrm{m}\left(\mathrm{PM}_{2.5}\right)$, and total suspended particles (TSP).

c Observed annual mean concentrations are calculated by averaging available monthly mean observations. For observations by Cao et al. (2007), January and July concentrations are averaged to calculate each annual mean.

d Simulated annual mean concentrations are calculated by averaging results from the months used to calculate the observed annual means at each site.

e [1] Qu et al. (2008); [2] Cao et al. (2009); [3] X. Y. Zhang et al. (2008); [4] Y. M. Han et al. (2008); [5] Cao et al. (2007).

${ }^{\mathrm{f}}$ At Muztagh Ata each filter sample was nominally collected over a one week period. However, during periods when the solar power supply was insufficient, the samples were regarded as valid and analyzed when the sampling standard air volume was larger than $30 \mathrm{~m}^{3}$ (Cao et al., 2009).

$\mathrm{g}$ Measurements were made at Muztagh Ata from Dec 2003 to Feb 2006. Only data from 2005 is used in this study.

${ }^{\mathrm{h}}$ Gucheng site is at a rural location but within an area of rapid urbanization according to X. Y. Zhang et al. (2008). Its EC and OC concentrations are very high and show obvious influence from local sources. We categorize it as an urban site for this study. 

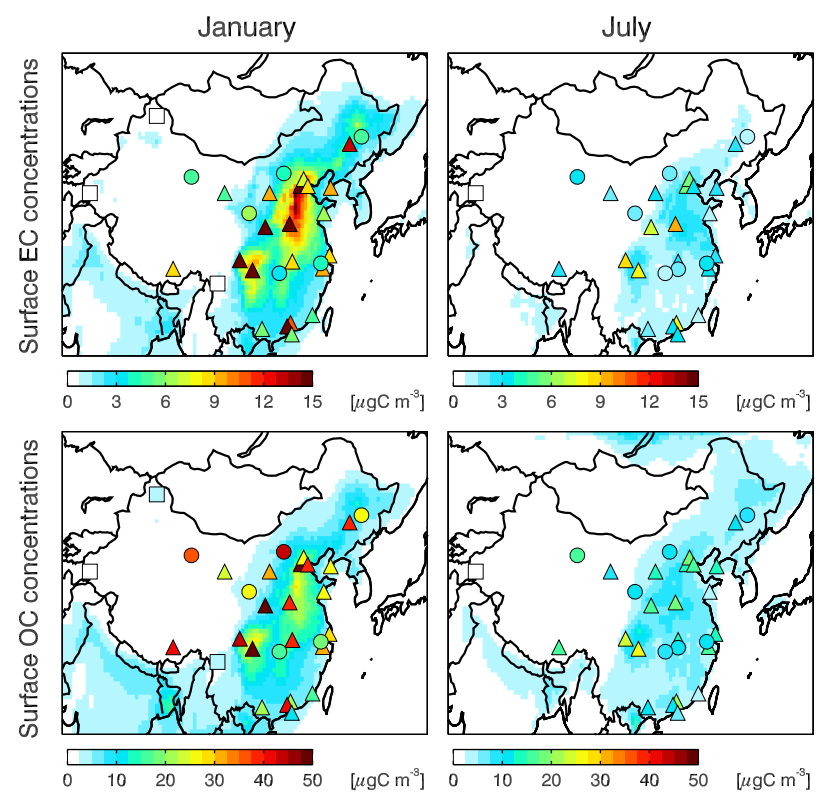

Fig. 5. Observed surface EC (top) and OC (bottom) concentrations in January and July at background (squares), rural (circles), and urban (triangles) Chinese sites. Underlain are simulated EC and OC monthly mean concentrations for January and July 2006 using bottom-up emission inventories.

by suppressing dry deposition when this happened, but we found the difference in monthly mean surface concentrations to be less than $5 \%$. In addition, our top-down emission estimates (Sect. 4) were derived using only measurements at non-urban (rural and background) locations, which are generally far from strong surface sources. We will return to the issue of model resolution in Sect. 6.

Figure 5 compares the spatial distributions of observed and simulated surface mean EC and OC concentrations for January and July. Highest concentrations were observed in the densely populated and industrialized areas of northern China, the Sichuan Basin, and the YRD and PRD megacity clusters. Two sites in western China (rural Dunhuang and urban Lasha) showed high concentrations in January. Urban sites were consistently more polluted than nearby rural sites, reflecting strong local emissions. At all sites where both January and July measurements were available, EC and OC concentrations were higher in winter than in summer, except at Panyu where the OC concentrations were similar. Although meteorological factors such as boundary layer compression in winter or enhanced wet deposition in summer may lead to such seasonal differences, we found that these effects are small based on the model analysis described below. Instead, stronger anthropogenic emission during the cold months was the main cause for the higher surface concentrations observed in winter.

The simulated EC and OC concentrations showed a westto-east gradient, reflecting the strong emissions in eastern
China in the bottom-up inventories, with highest concentrations over northern China and the Sichuan Basin. The model showed higher EC and OC concentrations in winter than in summer, similar to the observations. Understandably, the model was unable to resolve individual urban hotspots. However, the model also underestimated EC and OC concentration at almost all rural sites, particularly in January (with the exception of EC at Taiyangshan). This indicates a regionwide underestimate of carbonaceous aerosol sources associated with anthropogenic activities.

Figures 6 and 7 examine in detail the seasonal variations of surface EC and OC concentrations observed at the ten background and rural sites. At the two background sites in western China, Muztagh Ata and Akdala, EC and OC concentrations were significantly lower than those observed at other Chinese rural sites throughout the year. This indicates that foreign pollution transported from the west is not a significant contributor to the high levels of surface EC and OC observed at interior Chinese sites. Winter, summer, and fall measurements were also low at the Zhuzhang background site in southwestern China. However, data were missing for spring when the impact of transported biomass burning plumes from Southeast Asia to air quality in southern China may be greatest (Deng et al., 2008). Surface concentrations of both EC and OC were higher in fall and winter than in summer at most rural sites (except at Wusumu, where data were available for only four months), again suggesting strong region-wide emissions, likely associated with heating. Both EC and OC concentrations at Dunhuang and Gaolanshan were extremely high and showed large enhancements in fall and winter, suggesting that these sites are affected by local anthropogenic activities.

Figure 6 also shows the simulated surface EC concentrations using the bottom-up emission inventories. The simulation reproduced the relatively low concentrations observed at the three background sites. The model agreed well with summertime measurements at Taiyangshan, Jinsha, and Longfengshan, overestimated wintertime measurements at Taiyangshan, but otherwise underestimated observed rural values. This suggests that the EC emissions in the model are too low and misrepresented in space and time. Model concentrations were higher in winter than in summer, similar to the observations. Model performance was poorest at Dunhuang and Gaolanshan, where the bottom-up inventories had very little emissions. The average simulated annual mean EC concentration for all rural and background sites was $1.1 \mu \mathrm{gC} \mathrm{m}^{-3}, 56 \%$ lower than the observed $2.5 \mu \mathrm{gC} \mathrm{m}^{-3}$.

We further break down in Fig. 6 the contributions to simulated surface EC concentrations from Chinese anthropogenic residential and non-residential emissions, Chinese biomass burning emissions, and transport from foreign sources. Contributions from foreign sources were significant only at background sites, in particular at Zhuzhang in spring, which reflects the transport of biomass burning aerosols from Southeast Asia. Chinese biomass burning emissions have distinct 

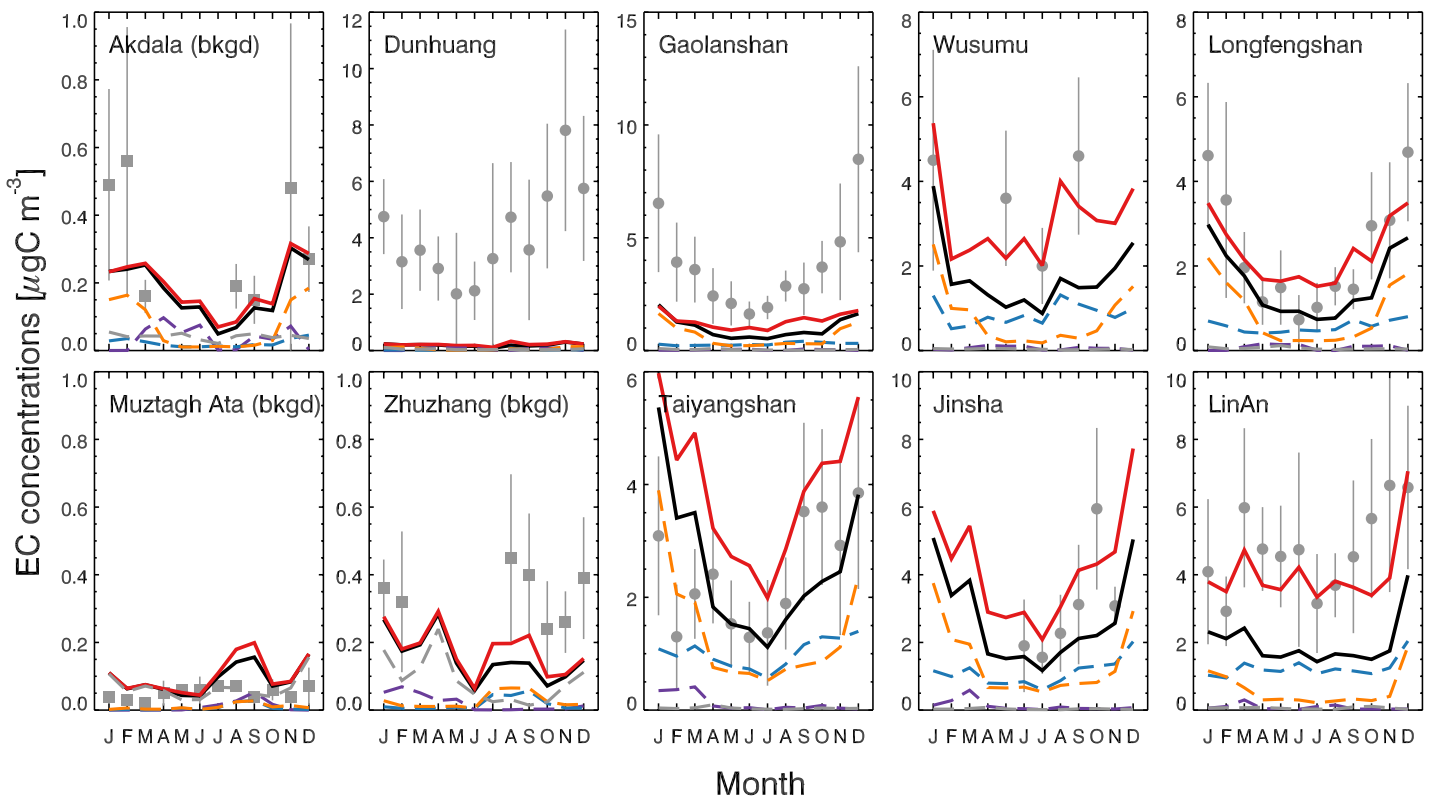

Fig. 6. Monthly mean surface EC concentrations observed at ten Chinese background (grey squares) and rural (grey circles) sites. Thin grey vertical lines indicate the standard deviations of the monthly mean observations. Solid lines indicate the simulated monthly mean concentrations using bottom-up emission inventories (black) and top-down emission estimates (red). Also shown are simulated components of surface EC concentrations: Chinese anthropogenic residential (orange dashed), Chinese anthropogenic non-residential (blue dashed), Chinese biomass burning (purple dashed), and contributions from non-Chinese sources (gray dashed).

seasonal patterns (Duncan et al., 2003; Song et al., 2010; Huang et al., 2012), but they have limited impacts at the seven rural sites as simulated by the model. The simulated EC concentrations at all seven rural sites were almost entirely due to Chinese anthropogenic residential and non-residential emissions. Residential emission had the most pronounced seasonal pattern in the model, decreasing rapidly by midspring and rising again in late fall, and it is the main driver for the seasonal variation in the simulated surface EC concentrations. Emissions from the non-residential sector in our model had a mild seasonal variation, and the simulated contributions of that sector likewise did not change much seasonally. Therefore, the observed higher concentrations in winter are unlikely to be due to meteorological factors alone, since these are accounted for in the model. Instead, the observed higher wintertime levels are mainly due to stronger emissions during the cold months, most likely associated with heating.

Figure 7 shows the seasonal variation of simulated surface OC concentrations. Again the model reproduced the low concentrations at background sites. The model severely underestimated measured OC concentrations at all rural sites year-round, except at Taiyangshan from January to March. Moreover, the model failed to reproduce the seasonal pattern at any of the rural sites. This suggests that primary emissions and secondary formation of OC are both poorly represented in the model. Model performance at Dunhuang and Gaolanshan was worse for OC than for EC, particularly during cold months, suggesting that a source associated with a high OC/EC emission ratio is missing in the bottom-up inventories. The average simulated annual mean $\mathrm{OC}$ concentration for all rural and background sites was $3.4 \mu \mathrm{gC} \mathrm{m}^{-3}$, $76 \%$ lower than the observed $14 \mu \mathrm{gC} \mathrm{m}^{-3}$.

Figure 7 also shows the individual simulated OC components, including OC from primary sectors, semi-volatile SOC, and dicarbonyl SOC. Similar to EC, OC from foreign sources were significant only at the three background sites. Chinese biomass burning constituted $25 \%$ of the simulated surface OC at Taiyangshan and Jinsha in March, but otherwise played a minor role in surface $\mathrm{OC}$ concentrations at the rural sites in all seasons. From late fall to early spring, the simulated surface OC concentrations were mainly due to residential emission. Non-residential emission was relatively less important to $\mathrm{OC}$ than to $\mathrm{EC}$, reflecting the lower $\mathrm{OC} / \mathrm{EC}$ emission ratio from that sector. The semi-volatile SOC and dicarbonyl SOC sources were comparable in magnitude. SOC production was largest from early summer to early fall, due to the combined effect of higher biogenic precursor emissions and stronger photochemistry in warmer seasons.

Model performance can be summarized by the regression slopes and correlations between model results and observations. Figures 8 and 9 show the scatter plots of simulated versus observed monthly mean surface EC and OC concentrations at rural and background sites for each season. Using the bottom-up inventories, the model underestimated EC observations in all seasons by $35 \%$ to $60 \%$ (reduced major axis 

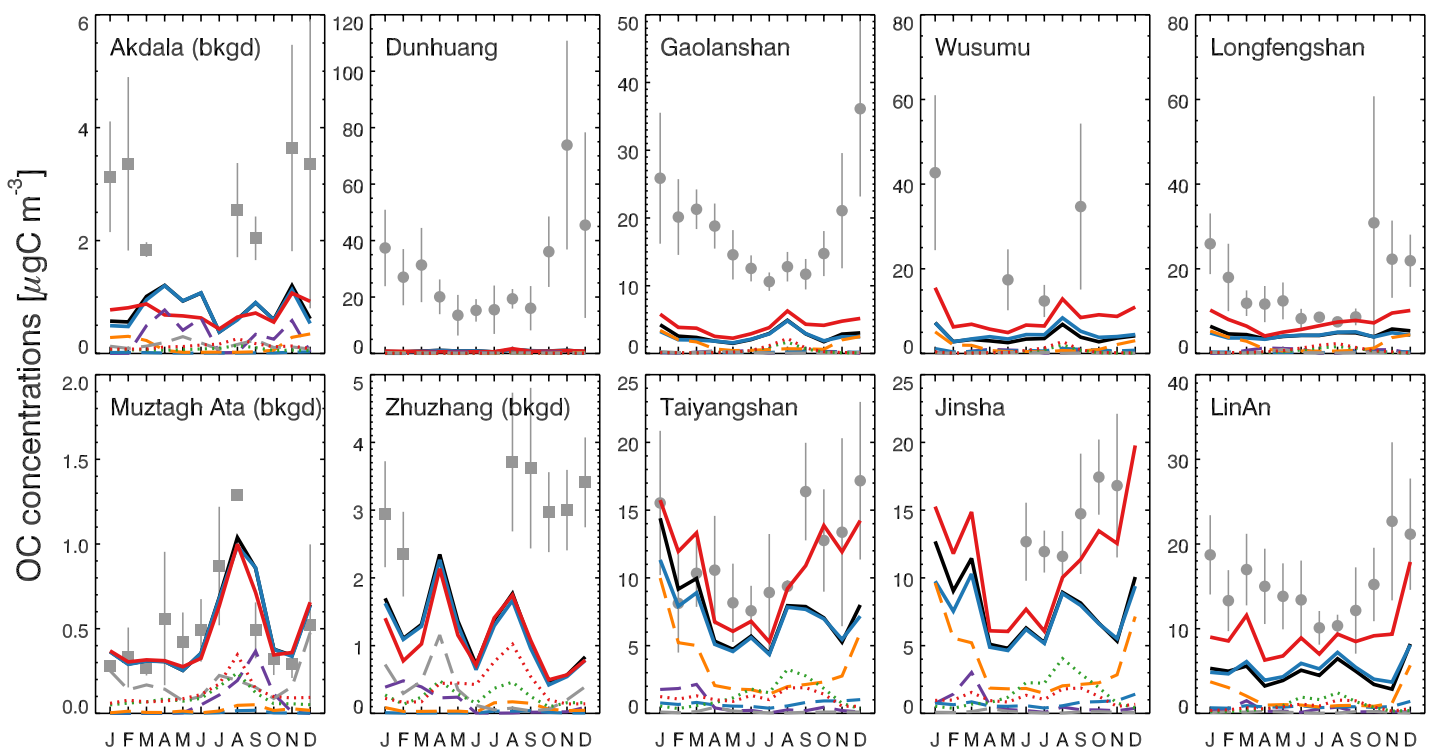

Month

Fig. 7. Monthly mean surface OC concentrations at ten Chinese background (grey squares) and rural (grey circles) sites. Thin grey vertical lines indicate the standard deviations of the monthly mean observations. Solid lines indicate simulated monthly mean concentrations using bottom-up emission inventories (black), top-down emission estimates (red), and hybrid emission estimates (blue). Also shown are simulated components of surface OC concentrations: Chinese anthropogenic residential (orange dashed), Chinese anthropogenic non-residential (blue dashed), Chinese biomass burning (purple dashed); semi-volatile SOC (green dotted), dicarbonyl SOC (red dotted), and contributions from non-Chinese sources (gray dashed).

regression slopes between 0.40 and 0.65 ), with correlation coefficients $r$ ranging from 0.41 to 0.48 . This again shows that the EC emissions in the model are underestimated, as well as misrepresented in space and time. For OC, the simulation using bottom-up inventories severely underestimated observed OC concentrations in all seasons, with regression slopes ranging from 0.16 to 0.51 . Moreover, the model could not capture the observed OC variability. The correlation coefficients $r$ ranged from 0.08 to 0.26 . This shows that (1) the bottom-up OC emissions are too low and misrepresented spatiotemporally, and (2) the representation of secondary formation in the model is poor. Model performance for OC was best in summer (slope $=0.51$ ), albeit with very poor correlation against observations $(r=0.26)$, because the combined contributions from primary emissions and secondary formation brought the model concentrations closer to observed values. For both EC and OC, the comparison of model results against observations was worst in fall. This may be because the model failed to capture the onset of enhanced anthropogenic emissions and/or the shut-down of biogenic SOC formation as the country moves into colder seasons. Also, there may be emission events in fall that are not currently represented in the model, for example, in-field burning of agricultural residues after the fall harvest (Yang et al., 2008).

Our analyses thus far show that Chinese surface OC concentrations at rural sites are strongly influenced by primary emissions, especially in winter. This is further illustrated in Fig. 10, which shows the correlations between monthly mean concentrations of $\mathrm{OC}$ and $\mathrm{EC}$ in the observation and in the model. Also shown are the reduced-major axis regression lines for OC versus EC measured at urban sites. At urban sites, measured $\mathrm{OC}$ and $\mathrm{EC}$ are tightly correlated in all seasons due to the overwhelming impact of local primary sources to surface concentrations. Even at rural and background sites, measured OC is still well correlated with EC in all seasons but especially in winter, indicating influence by primary emissions. We further note in Fig. 10 that the measured rural OC versus EC scatter to form "fan-like" patterns, and that the slopes of the lower-right edge of these "fans" are similar to the slopes of the measured urban OC versus EC regression lines. This suggests that Chinese rural and urban sites are affected by the same primary emission activities and thus have similar primary OC/EC emission ratios. The variability of rural OC away from the urban OC versus EC regression line reflects additional secondary production not represented in the model or primary OC sources not represented in the bottom-up inventories (e.g. at Dunhuang and Gaolanshan). 


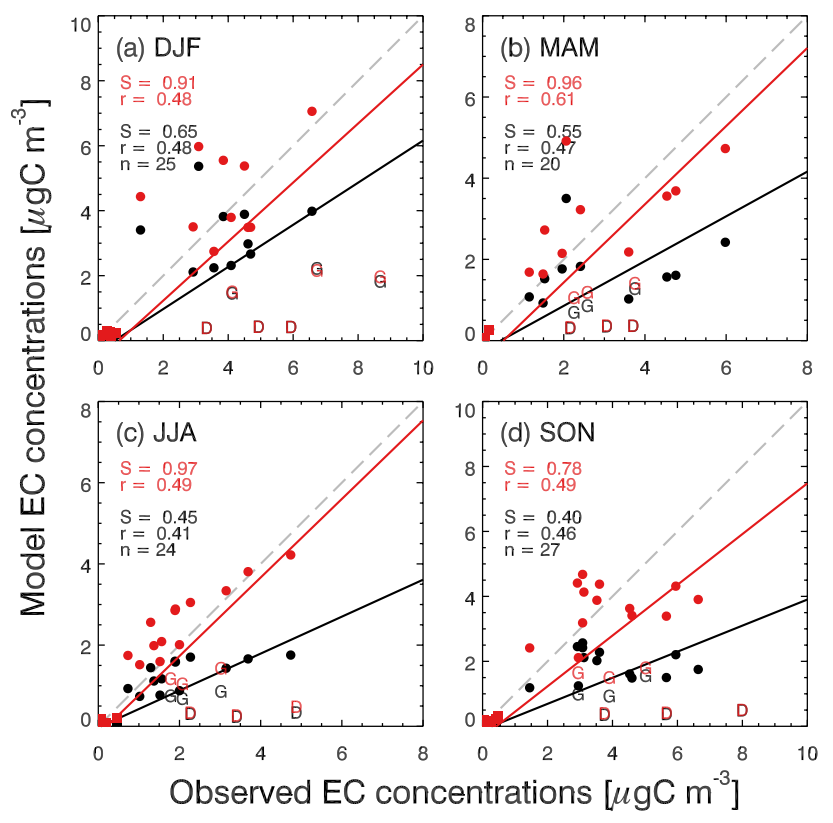

Fig. 8. Simulated versus observed monthly mean surface EC concentrations at rural (circles; Gaolanshan: G; Dunhuang: D) and background (squares) sites and the reduced-major axis regression lines (solid lines) for each season. Black: model results using bottom-up emission inventories; red: model results using top-down emission estimates. The reduced major-axis regression slope $(S)$ and correlation coefficient $(r)$ between model and observation, and number of data points $(n)$ for each season are shown inset. Grey dashed line indicate the 1:1 line.

\section{4 “Top-down" estimate of carbonaceous aerosol sources}

\subsection{EC emission estimates}

Based on the model evaluation presented in Sect. 3, we wished to know how the bottom-up EC and OC emissions should be adjusted to best reproduce the magnitude and spatiotemporal patterns of the observations. We used multiple regression to match the simulated EC contributions from each sector against monthly mean observations at the ten rural and background sites. We assumed that the simulated EC background (EC from non-Chinese sources) was correct, and subtracted it from the observations. The assumption of a correct EC background is valid, since the observed EC concentrations are low at Muztagh Ata and Akdala year-round, as well as at Zhuzhang in summer, fall, and winter, and the model reproduces these low concentrations. As previously mentioned, biomass burning in Southeast Asia in spring may affect air quality in southern China, but measurements were not available at Zhuzhang in spring to constrain this. The multiple regression model is

$$
\begin{gathered}
\boldsymbol{c}_{\mathrm{obs}}-\boldsymbol{c}_{\text {background }}=\beta_{1} \boldsymbol{c}_{\text {residential }}+\beta_{2} \boldsymbol{c}_{\text {non-residential }} \\
+\beta_{3} \boldsymbol{c}_{\text {biomass }}+\boldsymbol{\varepsilon} .
\end{gathered}
$$

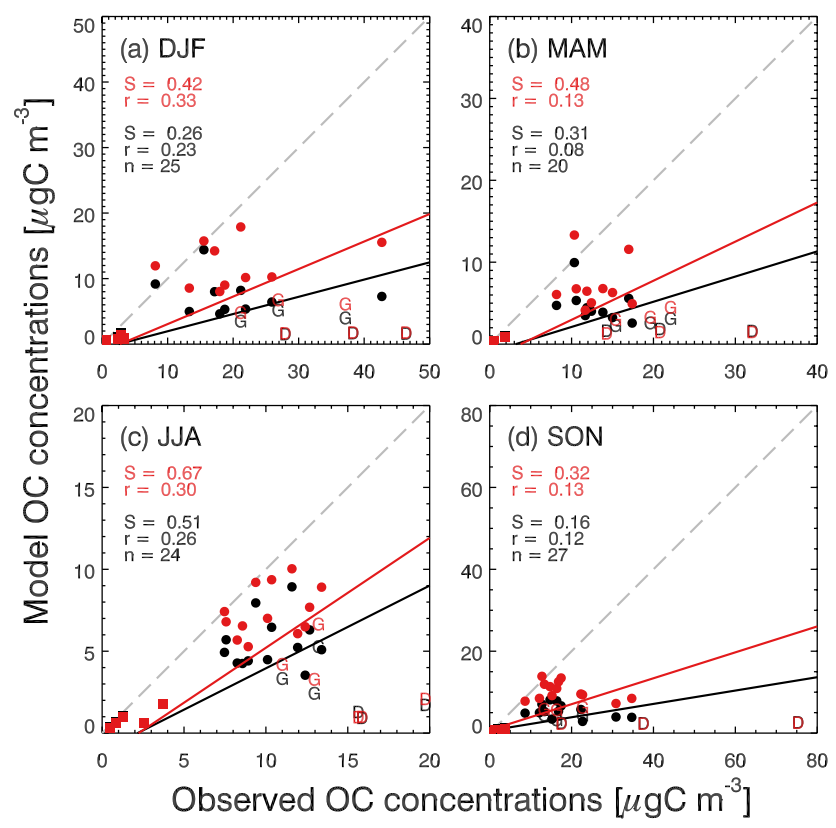

Fig. 9. Scatterplots of simulated versus observed monthly mean surface OC concentrations at rural (circles; Gaolanshan: G; Dunhuang: D) and background (squares) sites and the reduced-major axis regression lines (solid lines) for each season. Black: model results using bottom-up emission inventories; red: model results using topdown emission estimates. The reduced major-axis regression slope $(S)$ and correlation coefficient $(r)$ between model and observation, and number of data points $(n)$ for each season are shown inset. Grey dashed line indicate the 1:1 line.

In Eq. (1), $\boldsymbol{c}_{\mathrm{obs}}$ is the vector of observed monthly mean EC concentrations. $\boldsymbol{c}_{\text {background }}$ is the vector of simulated EC background from non-Chinese sources. $\boldsymbol{c}_{\text {residential }}$, $\boldsymbol{c}_{\text {non-residential }}$, and $\boldsymbol{c}_{\text {biomass }}$ are the vectors of simulated EC contributions from Chinese anthropogenic residential, anthropogenic non-residential, and biomass burning sources, respectively. $\varepsilon$ is the regression model error vector. $\beta_{1}$, $\beta_{2}$, and $\beta_{3}$ are the domain-wide scalar scale factors required for the source sectors to best match the observations as constrained by the multiple regression.

In practice, the contribution from Chinese biomass burning could not be reliably fitted using available data. This is seen in Fig. 6, where the observations do not reflect the seasonal signatures of biomass burning emissions. We conducted a lack-of-fit F-test and found that including the biomass burning component does not significantly improve the regression. Therefore, we also assumed Chinese biomass burning EC emission to be correct and removed its contribution prior to the regression. Since the rural EC measurements mostly reflected the spatiotemporal variability of anthropogenic residential and non-residential sources (Fig. 6), the estimates for these sources were not much affected.

The multiple regression gives a scale factor for the anthropogenic residential sector $\left(\beta_{1}\right)$ of 0.64 , with a 


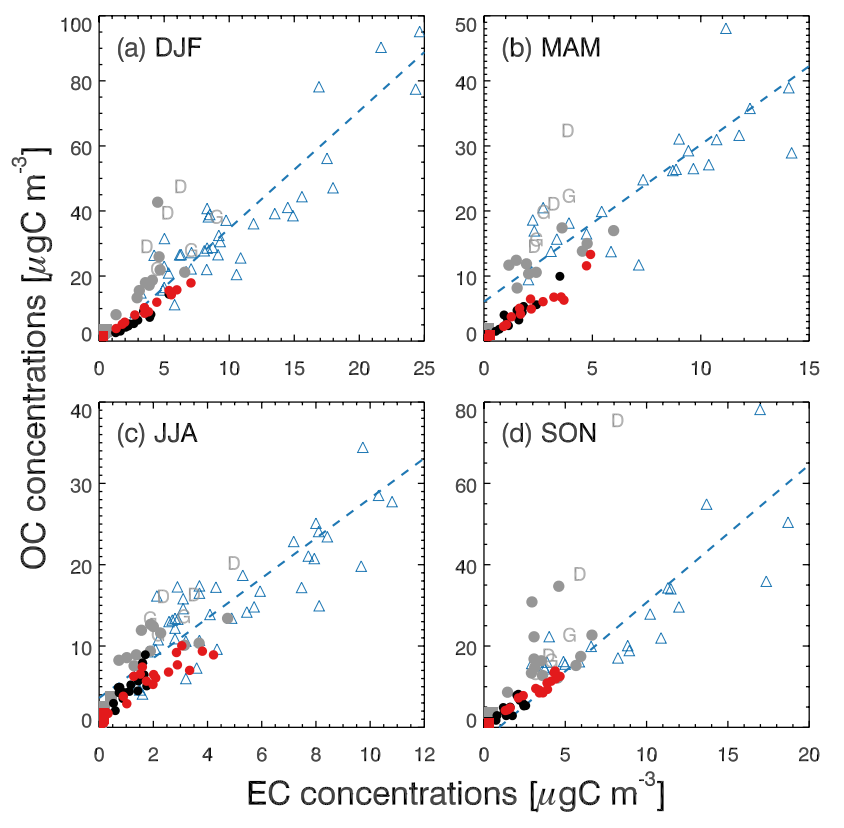

Fig. 10. OC versus EC concentrations observed (grey circles; G: Gaolanshan; D: Dunhuang) and simulated using bottom-up (black) and top-down (red) emissions at rural and background sites for each season. Also shown are observed OC versus EC concentrations (blue triangles) and reduced major-axis regression lines at urban sites (blue dashed lines) for each season.

standard deviation of \pm 0.49 (bootstrap estimation) and $p$ value $=0.08$. The scale factor for the anthropogenic nonresidential sector $\left(\beta_{2}\right)$ is 2.85 , with a standard deviation of \pm 0.42 and $p$-value $<0.001$. The multiple regression explains $59 \%$ of the observed variance (adjusted $R^{2}=0.59$ ).

It may seem surprising that the multiple regression indicates reduction of the residential emissions, given that the observations show a stronger wintertime EC enhancement compared to the simulation using bottom-up inventories. This is partly because the non-residential emissions are also slightly enhanced in winter, such that the combined effect of scaling the two anthropogenic sectors still increases the cold-month emissions in our top-down estimate. More importantly, the distinction between the residential and nonresidential sources in the multiple regression is somewhat arbitrary, because the spatial patterns of the residential and non-residential emissions are similar (Fig. 1). A better way of understanding the results is that the regression adjusts the two anthropogenic sectors simultaneously to best match both the annual mean concentrations and the cold-month enhancements in the observations. Thus only the total anthropogenic emission is meaningfully constrained.

Table 1 shows our top-down EC emission estimate. Total anthropogenic emission is $2.94 \mathrm{TgC}^{-1}$. Adding the unchanged biomass burning emission, the total Chinese EC emissions is $3.05{\mathrm{TgC} \mathrm{yr}^{-1}}^{-1} 59 \%$ larger from the bottom-up estimate. The standard deviation of the top-down EC emission estimate is $\pm 0.78 \mathrm{TgC} \mathrm{yr}^{-1}$, calculated by combining the top-down uncertainties (standard deviation of the scaling factors from the multiple regression calculated by bootstrapping) in quadrature and assuming the bottom-up uncertainty for biomass burning emissions. For comparison with previous bottom-up inventories (Table 1), we calculate the $95 \%$ confidence interval of our top-down emission estimate to be $\pm 50 \%$. This assumes that the emission estimate probabilities are normally distributed, but the true probabilities are more likely to be lognormally distributed (Streets et al., 2003a).

Figure 1 shows the spatial distribution of the top-down EC emission estimate. Compared to the bottom-up inventories, EC emissions are greatly enhanced in northern and central China, Sichuan basin, and over the YRD and PRD megacity clusters.

We conducted two sensitivity tests to evaluate the robustness of our multiple regression. First, we summed up $\boldsymbol{c}_{\text {residential }}$ and $\boldsymbol{c}_{\text {non-residential }}$ in Eq. (1) and fitted the observations against the combined anthropogenic contribution. The resulting estimate for the total anthropogenic EC emissions

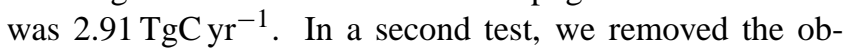
servations at Dunhuang and Gaolanshan from the multiple regression. The resulting estimate for total anthropogenic $\mathrm{EC}$ emissions was $2.58 \mathrm{TgC} \mathrm{yr}^{-1}$. Both estimates are within $12 \%$ of our original top-down estimate shown in Table 1.

\subsection{OC emission estimates}

Constraining OC emissions from filter-based OC measurements is challenging, because it is difficult to separate the primary and secondary OC. One way to circumvent this issue is to estimate $\mathrm{OC}$ emissions by scaling EC emissions with OC/EC emission ratios. We experimented with two methods for estimating $\mathrm{OC}$ emissions and summarize the results in Table 1 . Both methods provide traceability and can easily be recalculated when updated OC/EC emission ratios become available.

For the first method, we scaled the bottom-up anthropogenic OC emissions with the same coefficients $\beta_{1}$ and $\beta_{2}$ obtained from the multiple regression for anthropogenic EC emissions. This is equivalent to applying the OC/EC emission ratios used in the Zhang et al. (2009) bottom-up inventory, and hence can be considered as a "hybrid" approach, combining "bottom-up" statistics with "top-down" observational constraints. The resulting estimate for anthropogenic

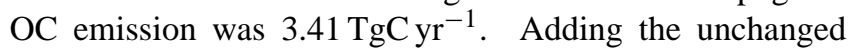
biomass burning emission estimate of $0.73 \mathrm{TgC} \mathrm{yr}^{-1}$, the total Chinese OC emission was estimated to be $4.14 \mathrm{TgC} \mathrm{yr}^{-1}$, only $5 \%$ greater than the bottom-up estimate with very similar spatial distribution (not shown). The uncertainty is similar to that of the bottom-up inventories.

A second, purely "top-down", approach for constraining OC emissions uses the primary OC/EC ratios derived from ambient observations. This method is legitimate because, 
as argued previously, the distinction between the residential and non-residential components in the EC multiple regression is not robust. Therefore, it is more appropriate to apply an overall primary OC/EC ratio representative of the ambient air mixture. Cao et al. (2007) defined the 20th percentile of observed OC/EC ratio as the primary OC/EC ratio for a particular location in a given season. It is important to distinguish primary OC/EC ratios for different seasons, because the relative strengths of source sectors can change throughout the course of a year. For northern cities $\left(>32^{\circ} \mathrm{N}\right)$, Cao et al. (2007) observed primary OC/EC ratios of 2.81 in winter and 1.99 in summer. For southern cities, their observed primary OC/EC ratios were 2.10 in winter and 1.29 in summer. We adopted these values for rural emissions since Fig. 10 shows that rural and urban air are affected by the same primary sources. We used the summer primary OC/EC ratios for April to September and the winter ratios for the rest of the year.

Our top-down estimate for Chinese anthropogenic and biomass burning OC sources are $6.42 \mathrm{TgC} \mathrm{yr}^{-1}$ and $0.25 \mathrm{TgC} \mathrm{yr}^{-1}$, respectively. Total $\mathrm{OC}$ emission is $6.67 \mathrm{TgC} \mathrm{yr}^{-1}, 69 \%$ higher than the bottom-up estimate. We calculated the top-down $\mathrm{OC}$ uncertainties from the variance of the product of two independent variables (top-down EC emissions and primary OC/EC ratios) (Goodman, 1960). We used two times the difference between the 20th percentile and the 5th percentile of observed OC/EC ratio from Cao et al. (2007) to represent the standard deviation of primary OC/EC ratio. Uncertainties from each sector were added in quadrature. The resulting overall standard deviation is \pm 1.30

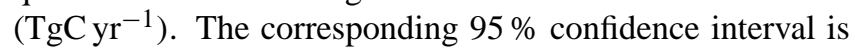
calculated as $\pm 38 \%$, again assuming normality for the emission estimate probabilities.

Figure 2 shows the spatial distributions of top-down OC emissions, which show large enhancements over northern and central China, Sichuan basin, as well as YRD and PRD megacity clusters, similar to the top-down EC emissions.

\subsection{Evaluation of the "top-down" emission estimates}

Figure 6 shows the simulated EC concentrations at the ten background and rural sites using the top-down EC emission estimates. Using the top-down emission estimates corrects the model low bias at Wusumu, Longfengshan, Jinsha, and LinAn, but the model now overestimates concentrations at Taiyangshan. The adjustments in Chinese emissions have little effect at the background sites. Overall, the simulated average annual mean surface EC concentrations at background and rural sites is improved to $1.9 \mu \mathrm{gC} \mathrm{m}{ }^{-3}$, in better agreement with observations $\left(2.5 \mu \mathrm{gC} \mathrm{m}^{-3}\right)$. The regression slopes for simulated versus observed EC concentrations are greatly improved in all seasons (Fig. 8), with best slope as high as 0.97 in summer, although the correlations are only slightly increased. As shown in Fig. 8, the remaining low bias relative to the observation is mostly due to the under- estimation at Dunhuang and Gaolanshan, where emissions are missing in the bottom-up inventories such that applying domain-wide scaling factors do not bring about a great improvement.

Figure 7 shows the model performance for OC at background and rural sites using the hybrid and top-down $\mathrm{OC}$ emission estimates. Applying the hybrid OC emission estimates does not lead to improvement in model performance, since it is very similar to the original bottom-up emissions. We will focus on the "pure top-down" OC emission estimates from now on.

Using top-down emission estimates, our simulated OC concentrations are improved vastly at Taiyangshan, Jinsha, and LinAn, especially in winter. However, the model is still biased low at Dunhuang, Gaolanshan, Wusumu, and Lonfengshan. Overall, the averaged simulated annual mean OC at background and rural sites increases to $5.4 \mu \mathrm{gC} \mathrm{m}{ }^{-3}$, although still $61 \%$ lower than observations $\left(14 \mu \mathrm{gC} \mathrm{m}{ }^{-3}\right)$. As shown in Fig. 9, the regression slopes for simulated versus observed OC are improved for all seasons, but the model remains unable to capture the variability in the observations. Again, this shows that both the primary emissions and the secondary formations of OC are poorly simulated in the model. Model low-bias is still worst in fall, because the model either fails to capture the onset of cold-month emissions or misses specific emission events. Neither of these shortcomings can be corrected by applying domainwide scaling factors.

Figure 11 shows the simulated components of annual mean surface OC using the top-down emission estimates. The national simulated annual mean surface OC is $4.5 \mu \mathrm{gC} \mathrm{m}^{-3}$. Primary anthropogenic emission is the dominant source of surface OC, contributing $3.2 \mu \mathrm{gC} \mathrm{m} \mathrm{m}^{-3}$ to the Chinese annual mean and accounting for over $60 \%$ of total OC over most of eastern China. Over western China, the simulated contribution of primary anthropogenic emission is generally less than $50 \%$, although in reality this is likely an underestimation due to missing OC emissions in our top-down estimates. Primary OC from Chinese biomass burning and non-Chinese sources each contributes less than $0.2 \mu \mathrm{gC} \mathrm{m}{ }^{-3}$ to the national annual mean.

Figure 10 shows the slopes of simulated OC versus EC concentrations in our model using the top-down emission estimates. The slopes of simulated non-urban OC versus EC are brought closer to (1) the slopes of observed OC versus $\mathrm{EC}$ at urban sites, and (2) the slopes of the lower-right edge of the data point "fans" formed by observed OC versus EC at non-urban sites. This indicates that the top-down estimates better represent the EC and OC emissions affecting Chinese urban and non-urban air, compared to the bottom-up inventories.

Even when using top-down emission estimates, the model still severely underestimates OC at rural and background sites year-round (Figs. 7 and 9). Excluding Dunhuang and Gaolanshan, we found that the remaining model OC low-bias 
(a) Anthropogenic primary OC

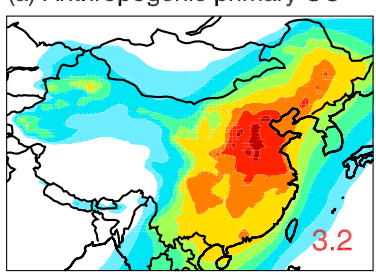

(d) Semi-volatile SOC

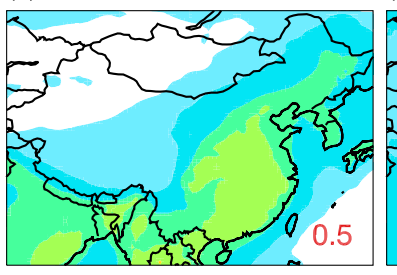

(b) Biomass burning primary OC

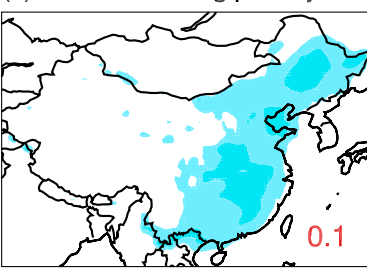

(e) Dicarbonyl SOC

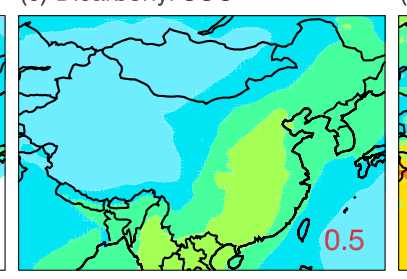

(c) Non-Chinese primary OC

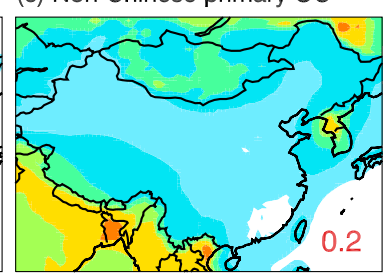

(f) Total OC

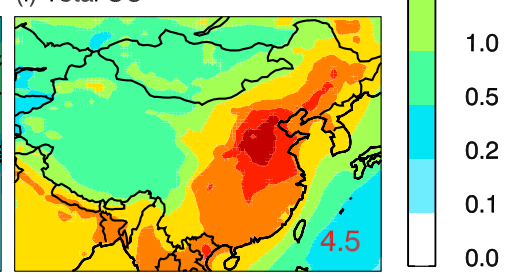

Fig. 11. Simulated components of surface annual mean OC using top-down emission estimates: (a) Chinese anthropogenic primary OC (including residential and non-residential sources); (b) Chinese biomass burning primary OC; (c) non-Chinese primary OC; (d) semi-volatile SOC from biogenic and anthropogenic VOC precursors; (e) SOC from irreversible uptake of dicarbonyls by aqueous-phase particles (mainly in clouds); and (f) total simulated OC. Simulated Chinese annual mean surface concentrations for each component are shown inset in red.

is not correlated with observed EC in summer $(r=0.39$, not significantly different from zero at $5 \%$ significance level, not shown). This suggests that the top-down inventory captures most of the primary OC emissions in eastern China in summer, and that the remaining model OC low-bias is likely due to missing SOC in the model. However, the model OC lowbias is significantly correlated with observed EC in eastern China for spring, fall, and winter, as well as in western China year-round (not shown). This suggests that the primary OC sources are still too low in our top-down estimate.

\section{Contribution of secondary formation to $\mathrm{OC}$}

Finally, we quantified the contribution of secondary formation to surface OC. As shown in Fig. 11, semi-volatile SOC and dicarbonyl SOC each contributes $0.5 \mu \mathrm{gC} \mathrm{m}^{-3}$ to the Chinese annual mean surface OC in our model. SOC from both formation pathways are highest over southeastern China, reflecting the combined impact of high precursor emissions and strong photochemistry. On an annual basis, SOC accounts for $21 \%$ and $19 \%$ of simulated surface OC for the whole of China and in eastern China, respectively. In summer, the simulated secondary fraction of surface OC is $47 \%$ for the whole of China and $45 \%$ in eastern China. This is likely an underestimation, because our simulated OC concentrations are lower than observations (Figs. 7 and 9) in summer. We mentioned previously that the remaining OC differences between the top-down model results and observations at Longfengshan, Taiyangshan, Jinsha, Wusumu, and LinAn in summer are not correlated with observed EC. As an upper estimate, we assumed that these remaining OC differences are entirely secondary, thus estimated that $62 \%$ of observed rural surface OC is due to secondary formation in eastern China in summer.

Table 2 summarizes the simulated SOC produced by individual VOC precursors in China. The most important precursor is isoprene, followed by monoterpenes and aromatics, accounting for $39 \%, 23 \%$, and $17 \%$ of total simulated $\mathrm{SOC}$, respectively. In terms of source types, biogenic VOC is the largest source of SOC in our model, accounting for $70 \%$ of total simulated SOC. Anthropogenic and biomass burning VOC accounts for $24 \%$ and $6 \%$ of total simulated SOC, respectively. Our findings differ from the results of Z. Han et al. (2008), who found monoterpenes to be the largest sources of SOC in summer in China. This is partly because we used larger isoprene emissions based on satellite formaldehyde observations (Fu et al., 2007). More importantly, we included an additional SOA formation pathway via aqueous uptake of dicarbonyls, of which isoprene is the dominant source. As a result, our simulated SOC concentrations in summer over eastern China are approximately twice their simulated values, and our simulated total OC concentrations are in better agreement with observations at the ten rural and background sites.

\section{Comparison with previous studies}

Table 4 compares our top-down estimates for Chinese anthropogenic carbonaceous aerosol emissions with previous bottom-up inventories for recent years. Previous bottomup estimates of Chinese anthropogenic emissions range from $0.94 \mathrm{TgC} \mathrm{yr}^{-1}$ to $1.81 \mathrm{TgC} \mathrm{yr}^{-1}$ for $\mathrm{EC}$ and from $2.41 \mathrm{TgC} \mathrm{yr}^{-1}$ to $3.84 \mathrm{TgC} \mathrm{yr}^{-1}$ for OC. Our estimates for both EC and OC are at least $60 \%$ larger than all previous 
Table 4. Estimates of Chinese anthropogenic carbonaceous aerosol emissions after the year 2000.

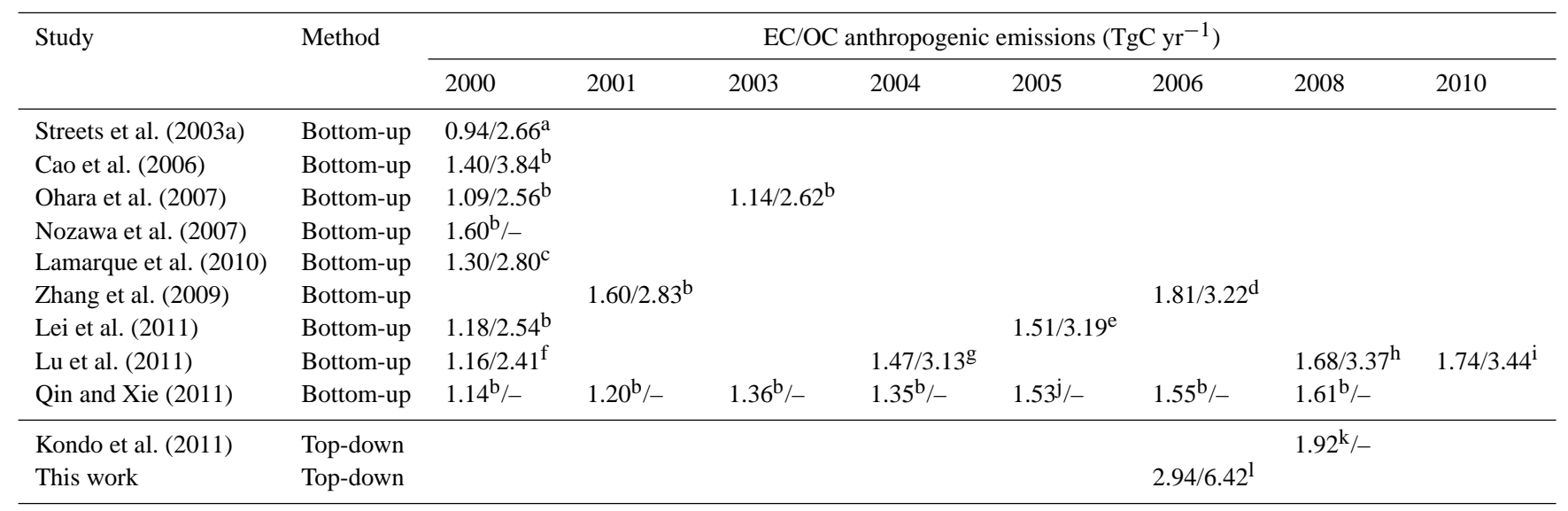

${ }^{a}$ Uncertainties, represented as $95 \%$ confidence intervals (CI), were $\pm 484 \%$ and $\pm 495 \%$ for EC and OC, respectively.

${ }^{\mathrm{b}}$ Uncertainties not reported in paper.

${ }^{\mathrm{c}}$ Chinese anthropogenic EC and OC emissions taken from Bond et al. (2007) with updates from T. Bond (personal communication, 2011). Uncertainties were expected to be larger than a factor of 2 .

${ }^{\mathrm{d}}$ Uncertainties (represented as $95 \% \mathrm{CI}$ ) were $\pm 208 \%$ for $\mathrm{EC}$ and $\pm 258 \%$ for OC, respectively.

e Uncertainties (represented as $95 \%$ CI) were $\pm 187 \%$ and $\pm 229 \%$ for EC and OC, respectively.

${ }^{\mathrm{f}}$ Uncertainties (represented as $95 \% \mathrm{CI}$ ) were $0.62-2.29 \mathrm{TgC} \mathrm{yr}^{-1}$ for $\mathrm{EC}$ and $1.22-4.70 \mathrm{TgC} \mathrm{yr}^{-1}$ for OC.

$\mathrm{g}$ Uncertainties (represented as $95 \% \mathrm{CI}$ ) were $0.80-2.98 \mathrm{TgC} \mathrm{yr}^{-1}$ for $\mathrm{EC}$ and $1.57-6.22 \mathrm{TgC}^{-1}$ for OC.

${ }^{\mathrm{h}}$ Uncertainties (represented as $95 \% \mathrm{CI}$ ) were $0.95-3.17 \mathrm{TgC} \mathrm{yr}^{-1}$ for EC and 1.73-6.57 $\mathrm{TgC} \mathrm{yr}^{-1}$ for OC.

${ }^{\mathrm{i}}$ Uncertainties (represented as $95 \% \mathrm{CI}$ ) were $0.97-3.31 \mathrm{TgC} \mathrm{yr}^{-1}$ for EC and $1.73-6.78 \mathrm{TgC}^{-1}$ for OC. $^{-}$

$\mathrm{j}$ Total Chinese $\mathrm{EC}$ emission, including $0.04 \mathrm{TgC} \mathrm{yr}^{-1}$ from biomass burning sources, is $1.57 \mathrm{TgC}^{-1}$. Uncertainty (represented as $95 \% \mathrm{CI}$ ) for total Chinese EC emission was

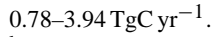

${ }^{\mathrm{k}}$ Top-down BC emission estimate based on measurements at Cape Hedo Observatory, Japan from February 2008 to May 2009 . The estimated uncertainty is $40 \%$.

${ }^{1}$ Uncertainties, represented as standard deviations, are $\pm 0.60 \mathrm{TgC}^{-1}$ for $\mathrm{EC}$ and $\pm 1.10 \mathrm{TgC} \mathrm{yr}^{-1}$ for OC. The corresponding $95 \% \mathrm{CI}$ (calculated assuming normal distribution) are $\pm 40 \%$ for EC and $\pm 34 \%$ for OC, respectively. See text for details.

bottom-up inventories (Streets et al., 2003a; Bond et al., 2007; Zhang et al., 2009; Lamarque et al., 2010; Lei et al., 2011; Lu et al., 2011; Qin and Xie, 2011). In particular, our emission estimates for the year 2006 for both EC and OC are more than $120 \%$ higher than the emission estimates developed by Lamarque et al. (2010) for the year 2000, which were used in the Coupled Model Intercomparison Project Phase 5 (CMIP5). Our top-down emission estimates for both EC and OC for the year 2006 are within the range of uncertainties reported by bottom-up inventories for the years 2005 to 2010 (Zhang et al., 2009; Lei et al., 2011; Lu et al., 2011; Qin and Xie 2011).

Why are previous bottom-up estimates for Chinese carbonaceous aerosol emissions so similarly low? One possibility is that because bottom-up inventories are mostly based on activity statistics from the same data sources, under-reporting of activity levels and/or over-reporting of emission control implementations would affect all bottom-up estimates in a similar manner. Another reason may be because emission factors specific to Chinese conditions are scarcely measured and highly variable. Researchers, out of necessity, often adopt middle-of-the-road emission factors from the literature, mostly measured in Western societies (Streets et al., 2001, 2003a; Bond et al., 2004; Cao et al., 2006; Ohara et al., 2007; Zhang et al., 2009; Lei et al., 2011; Lu et al., 2011;
Qin and Xie, 2011; and references therein). In particular, the emission factors from residential combustion can vary by an order of magnitude depending on the share of low- versus high-quality fuel (e.g. briquettes versus raw coal) (Zhang et al., 2009; Lu et al., 2011), but there is currently no good statistics for that information. As a result, the median estimates from previous bottom-up inventories are all similarly low, while the associated uncertainties are all similarly large.

Our top-down estimate for OC emission from biomass burning is $0.25 \mathrm{TgC} \mathrm{yr}^{-1}$, lower than the bottom-up inventory of Streets et al. (2003b). This is because the overall primary OC/EC ratios we adopted from Cao et al. (2007) represent urban air mixtures. Measured OC/EC emission ratios from biomass burning are generally much higher (Andreae and Merlet, 2001), so our estimate is likely to be low, although measurements are not available to constrain this source in the present study.

Our conclusion that Chinese anthropogenic carbonaceous aerosol emissions are underestimated is consistent with the studies of Z. Han et al. (2008) and Koch et al. (2009). Using aircraft measurements conducted downwind from China in April 2001, Hakami et al. (2005) found anthropogenic EC emissions from northern and northeastern China to be low by a factor of 2, and their derived emissions are consistent with our results. However, they found anthropogenic 
EC emissions from southeastern China to be overestimated by a factor of 2 , inconsistent with our findings. Kondo et al. (2011) measured BC concentration on a remote island in East China Sea and selectively analysed data strongly influenced by transport from China (mostly in spring and winter of the years 2008 and 2009). They concluded that Chinese annual anthropogenic $\mathrm{BC}$ emission is $1.92 \mathrm{TgC} \mathrm{yr}^{-1}$, similar to the $1.81 \mathrm{TgC} \mathrm{yr}^{-1}$ estimated by Zhang et al. (2009). Both Hakami et al. (2005) and Kondo et al. (2011) used observations far downwind from China during particular seasons, thus they may not reflect the total annual emissions from the entire country.

Matsui et al. (2009) simulated EC and OC in northern China in summer 2006 using anthropogenic emissions similar to those estimated by Zhang et al. (2009). They found that their simulated surface EC concentrations were too high at one urban site and one suburban site in Beijing, and accordingly concluded that EC emissions are overestimated around Beijing. Their EC measurements showed a strong diurnal cycle, which they attributed to the accumulation of pollutants near the surface due to nighttime boundary layer compression. They pointed out that a model with coarser vertical resolution would not be able to resolve this nighttime accumulation and would under-simulate the 24-h average EC concentrations even if the emissions were correct.

Our purpose in this study was to constrain Chinese annual carbonaceous aerosol sources on a national scale. We made no attempt to resolve urban pollution, which would be strongly influenced by local fluxes. Our top-down emission estimates are derived from rural and background measurements, which are less affected by local emissions and more representative of regional pollutant levels. At Dunhuang and Gaolanshan, where observations are clearly affected by local emissions in winter, the difference between model results and observations are too large to be explained by the coarseness of model resolution alone. Also, excluding the data from these two sites has little impact on our top-down emission estimates (Sect. 4.1).

Our top-down emission estimates do lead to high biases at Taiyangshan in central China. This indicates that the emissions in southern China may be biased high in our top-down estimate, potentially consistent with the findings of Hakami et al. (2005). However, the overall annual mean performance on a national scale is still much improved compared to that of the bottom-up inventory. This highlights the critical point that top-down emission estimates can only be derived from measurements that fully represent the spatiotemporal variability for the domain and resolution of interest.

\section{Conclusions}

We simulated carbonaceous aerosols in China and compared model results to surface measurements, with the goal of deriving top-down emission estimates of EC and
OC for China, as well as better quantifying the secondary sources of OC. We conducted a year-long simulation, driven by the current Chinese bottom-up emission inventories for EC $\left(1.92 \mathrm{TgC} \mathrm{yr}^{-1}\right)$ and OC $\left(3.95 \mathrm{TgC}^{-1}\right)$. The model contains updated SOC formation mechanisms, including reversible-partitioning of semi-volatile oxidation products from biogenic and anthropogenic VOC precursors, as well as reactive uptake of dicarbonyls on aqueous particles.

A database of surface measurements was compiled from previous studies to represent the spatial and seasonal variability of carbonaceous aerosols in China. Analyses of the observations show that surface EC and OC at Chinese urban and non-urban sites are strongly affected by anthropogenic emissions.

The comparison of model results to observations at background and rural sites evaluates the bottom-up inventories. The simulated average annual mean EC concentration for all rural and background sites is $1.1 \mathrm{\mu gC} \mathrm{m}^{-3}, 56 \%$ lower than the observed $2.5 \mu \mathrm{gC} \mathrm{m}^{-3}$. For OC, the model severely underestimates observed values at all sites year-round, with a simulated average annual mean $\mathrm{OC}$ concentration for rural and background sites of $3.4 \mu \mathrm{gC} \mathrm{m}{ }^{-3}, 76 \%$ lower than the observed $14 \mu \mathrm{gC} \mathrm{m}{ }^{-3}$. Moreover, the model fails to capture the spatiotemporal variability in the OC observations. This indicates that not only are the bottom-up OC emissions too low and spatiotemporally misrepresented, but that the representation of secondary formation in the model is also poor. The model grossly underestimates EC and OC at Dunhuang and Gaolanshan, where an anthropogenic source associated with high OC/EC emission ratio is missing in the bottom-up inventories. Model performances for EC and OC are both worst in fall. Sensitivity simulations show that meteorological factors and uncertainties in the aerosol removal rates cannot explain the low biases in the simulated concentrations. This suggests an underestimation of carbonaceous aerosol sources on a national scale.

Our use of multiple regression derives top-down emission estimates of EC based on monthly observations at rural and background sites. Only the total Chinese anthropogenic emission could be robustly constrained given the available observations. The resulting top-down estimate for Chinese EC emissions is $3.05 \pm 0.78 \mathrm{TgC} \mathrm{yr}^{-1}$.

We experimented with two approaches for scaling the top-down EC emissions with OC/EC emission ratios to derive $\mathrm{OC}$ emission estimates. A "hybrid" approach using the OC/EC emission ratios from the bottom-up inventory of Zhang et al. (2009) yields an estimate of total Chinese OC emission of $4.14 \mathrm{TgC} \mathrm{yr}^{-1}$, but applying this emission estimate does not significantly improve model performance. A second, purely "top-down", approach uses the primary $\mathrm{OC} / \mathrm{EC}$ ratios derived from ambient observations by Cao et al. (2007). The resulting top-down estimate for total Chinese $\mathrm{OC}$ emission is $6.67 \pm 1.30 \mathrm{TgC}^{-1}$.

Applying the top-down emission estimates, the simulated average annual mean concentrations at rural and background 
sites are improved to $1.9 \mu \mathrm{gC} \mathrm{m}{ }^{-3}$ (EC) and $5.4 \mu \mathrm{gC} \mathrm{m}{ }^{-3}$ (OC). For EC, the remaining low-bias relative to the observation is mostly due to missing emissions at Dunhuang and Gaolanshan. Even when using the top-down emission estimates, the model is still significantly underestimating observed OC concentrations in all seasons, with little skill in capturing the variability. We find that the top-down inventory is capturing most of the primary $\mathrm{OC}$ emissions in eastern China in summer. However, the primary OC sources are still too low in our top-down estimate in eastern China for all other seasons and in western China year-round.

Secondary formation accounts for $21 \%$ of Chinese annual mean surface OC in our model. In summer, as high as $62 \%$ of the observed surface OC may be due to secondary formation in eastern China. SOC produced from semi-volatile compounds and dicarbonyls are comparable in magnitude. The most important SOC precursor is isoprene, followed by monoterpenes and aromatics.

In summary, our analysis points to four shortcomings in the current bottom-up inventories of Chinese carbonaceous aerosols. Firstly, the anthropogenic source is underestimated on a national scale, particularly for $\mathrm{OC}$, likely due to uncertainties in emissions from small industries, residential combustion, and transportation (Zhang et al., 2009; Lu et al., 2011; Qin and Xie, 2011). Secondly, the spatial and temporal distributions of emissions are incorrect in the current bottom-up inventories. Thirdly, there is a missing source in western China, characterized by a high OC/EC emission ratio and a strong enhancement during the colder months, likely associated with the use of biofuels or other low-quality fuels for heating. The fourth issue is that sources in fall are not well represented, either because the seasonal shifting of emissions and/or secondary formation is poorly captured or because specific fall emission events are missing. In addition, the seasonal contributions from biomass burning in China and in Southeast Asia may also be underestimated, but measurements are not available to constrain these sources in this study. Our analyses are based on surface measurements. Aircraft observations of EC and OC concentrations may provide additional constraints on the contribution of foreign sources. Secondary production of $\mathrm{OC}$ is also underestimated in our model, either due to error in precursor emissions or missing secondary formation pathways.

Our top-down EC and OC emission estimates are at least $60 \%$ higher than all previous bottom-up estimates. Two questions arise. The first question is why are all previous bottom-up estimates so similarly low? We speculate that the use of activity statistics from the same data sources and the adoption of emission factors from the literature may be the cause. The second question is whether our emission estimates are supported by other observations? Our results are consistent with the findings from a few previous seasonal studies. We plan to explore the use of other data, such as satellite aerosol optical depth observations, to evaluate our results. Many gaps remain in our understanding of Chinese carbonaceous aerosol sources. More regional measurements with better spatiotemporal coverage, especially over western China, are needed to address these gaps.

Acknowledgements. This work is funded by the Research Grants Council of Hong Kong (PolyU 5175/09E) and by Peking University. We thank Shuhua Liu and Hongsheng Zhang for helpful discussions.

Edited by: E. Vignati

\section{References}

Andreae, M. O. and Merlet, P.: Emission of trace gases and aerosols from biomass burning, Global Biogeochem. Cy., 15, 955-966, doi:10.1029/2000GB001382, 2001.

Bey, I., Jacob, D. J., Yantosca, R. M., Logan, J. A., Field, B., Fiore, A. M., Li, Q., Liu, H., Mickley, L. J., and Schultz, M.: Global modeling of tropospheric chemistry with assimilated meteorology: model description and evaluation, J. Geophys. Res., 106, 23073-23096, 2001.

Bond, T. C., Streets, D. G., Yarber, K. F., Nelson, S. M., Woo, J.H., and Klimont, Z.: A technology-based global inventory of black and organic carbon emissions from combustion, J. Geophys. Res., 109, D14203, doi:10.1029/2003JD003697, 2004.

Bond, T. C., Bhardwaj, E., Dong, R., Jogani, R., Jung, S., Roden, C., Streets, D. G., and Trautmann, N. M.: Historical emissions of black and organic carbon aerosol from energy-related combustion, Global Biogeochem. Cy., 21, GB2018, 1850-2000, doi:10.1029/2006GB002840, 2007.

Cao, G., Zhang, X., and Zheng, F.: Inventory of black carbon and organic carbon emissions from China, Atmos. Environ., 40, 6516-6527, doi:10.1016/j.atmosenv.2006.05.070, 2006.

Cao, J. J., Lee, S. C., Chow, J. C. Watson, J. G., Ho, K. F., Zhang, R. J., Jin, Z. D., Shen, Z. X., Chen, G. C., Kang, Y. M., Zou, S. C., Zhang, L. Z., Qi, S. H., Dai, M. H., Cheng, Y., and $\mathrm{Hu}, \mathrm{K}$.: Spatial and seasonal distributions of carbonaceous aerosols over China, J. Geophys. Res., 112, D22S11, doi:10.1029/2006JD008205, 2007.

Cao, J. J., Xu, B. Q., He, J. Q., Liu, X. Q., Han, Y. M., Wang, G. H., and Zhu, C. S.: Concentrations, seasonal variations, and transport of carbonaceous aerosol at a remote Mountainous region in western China, Atmos. Environ., 43, 4444-4452, doi:10.1016/j.atmosenv.2009.06.023, 2009.

Carlton, A. G., Wiedinmyer, C., and Kroll, J. H.: A review of Secondary Organic Aerosol (SOA) formation from isoprene, Atmos. Chem. Phys., 9, 4987-5005, doi:10.5194/acp-9-4987-2009, 2009.

Chen, D., Wang, Y., McElroy, M. B., He, K., Yantosca, R. M., and Le Sager, P.: Regional CO pollution and export in China simulated by the high-resolution nested-grid GEOS-Chem model, Atmos. Chem. Phys., 9, 3825-3839, doi:10.5194/acp-9-3825-2009, 2009.

Chow, J. C., Watson, J. G., Pritchett, L. C., Pierson, W. R., Frazier, C. A., and Purcell, P. G.: The DRI thermal/optical reflectance carbon analysis system: Description, evaluation and applications in US air quality studies, Atmos. Environ., 27, 1185-1201, 1993.

Chow, J. C., Watson, J. G., Chen, L.-W. A., Arnott, W. P., Moosmüller, H., and Fung, K. K.: Equivalence of elemental 
carbon by thermal/optical reflectance and transmittance with different temperature protocols, Environ. Sci. Technol., 38, 44144422, 2004.

Chung, S. H. and Seinfeld, J. H.: Global distribution and climate forcing of carbonaceous aerosols, J. Geophys. Res., 107, 4407, doi:10.1029/2001JD001397, 2002.

Cooke, W. F., Liousse, C., Cachier, H., and Feichter, J.: Construction of a $1^{\circ} \times 1^{\circ}$ fossil fuel emission data set for carbonaceous aerosol and implementation and radiative impact in the ECHAM4 model, J. Geophys. Res., 104, 22137-22162, 1999.

De Haan, D. O., Hawkins, L. N., Kononenko, J. A., Turley, J. J., Corrigan, A. L., Tolbert, M. A., and Jimenez, J. L.: Formation of nitrogen-containing oligomers by methylglyoxal and amines in simulated evaporating cloud droplets, Environ. Sci. Tech., 45, 984-991, doi:10.1021/es102933x, 2011.

Deng, X., Tie, X., Zhou, X., Wu, D., Zhong, L., Tan, H., Li, F., Huang, X., Bi, X., and Deng, T.: Effects of Southeast Asia biomass burning on aerosols and ozone concentrations over the Pearl River Delta (PRD) Region, Atmos. Env., 42, 8493-8501, doi:10.1016/j.atmosenv.2008.08.013, 2008.

Duncan, B. N., Martin, R. V., Staudt, A. C., Yevich, R., and Logan, J. A.: Interannual and seasonal variability of biomass burning emissions constrained by satellite observations, J. Geophys. Res., 104, 4100, doi:10.1029/2002JD002378, 2003.

Fu, T.-M., Jacob, D. J., Palmer, P. I., Chance, K., Wang, Y. X., Barletta, B., Blake, D. R., Stanton, J. C., and Pilling, M. J.: Spaced-based formaldehyde measurements as constraints on volatile organic compound emissions in east and south Asia and implications for ozone, J. Geophys. Res., 112, D06312, doi:10.1029/2006JD007853, 2007.

Fu, T.-M., Jacob, D. J., Wittrock, F., Burrows, J. P., Vrekoussis, M., and Henze, D. K.: Global budgets of atmospheric glyoxal and methylglyoxal, and implications for formation of secondary organic aerosols, J. Geophys. Res., 113, D15303, doi:10.1029/2007JD009505, 2008.

Fu, T.-M., Jacob, D. J., and Heald, C. L.: Aqueous-phase reactive uptake of dicarbonyls as a source of organic aerosol over eastern North America, Atmos. Environ., 43, 1814-1822, doi:10.1016/j.atmosenv.2008.12.029, 2009.

Galloway, M. M., Chhabra, P. S., Chan, A. W. H., Surratt, J. D., Flagan, R. C., Seinfeld, J. H., and Keutsch, F. N.: Glyoxal uptake on ammonium sulphate seed aerosol: reaction products and reversibility of uptake under dark and irradiated conditions, Atmos. Chem. Phys., 9, 3331-3345, doi:10.5194/acp-9-3331-2009, 2009.

Goldstein, A. H., Fan, S. M., Goulden, M. L., Munger, J. W., and Wofsy, S. C.: Emissions of ethane, propene, and 1-butene by a midlatitude forest, J. Geophys., Res., 101, 9149-9157, 1996.

Goodman, L. A.: On the exact variance of products, J. American. Stat. Assoc., 55, 708-713, 1960.

Guenther, A., Hewitt, C. N., Erickson, D., Fall, R., Geron, C., Graedel, T., Harley, P., Klinger, L., Lerdau, M., McKay, W., Pierce, T., Scholes, B., Steinbrecher, R., Tallamraju, R., Taylor, J., and Zimmerman, P.: A global model of natural volatile organic compound emissions, J. Geophys. Res., 100, 8873-8892, 1995.

Guenther, A., Karl, T., Harley, P., Wiedinmyer, C., Palmer, P. I., and Geron, C.: Estimates of global terrestrial isoprene emissions using MEGAN (Model of Emissions of Gases and Aerosols from
Nature), Atmos. Chem. Phys., 6, 3181-3210, doi:10.5194/acp-63181-2006, 2006.

Hakami, A., Henze, D. K., Seinfeld, J. H., Chai, T., Tang, Y., Carmichael, G. R., and Sandu, A.: Adjoint inverse modeling of black carbon during the Asian Pacific Regional Aerosol Characterization Experiment, J. Geophys. Res., 110, D14301, doi:10.1029/2004JD005671, 2005.

Hallquist, M., Wenger, J. C., Baltensperger, U., Rudich, Y., Simpson, D., Claeys, M., Dommen, J., Donahue, N. M., George, C., Goldstein, A. H., Hamilton, J. F., Herrmann, H., Hoffmann, T., Iinuma, Y., Jang, M., Jenkin, M. E., Jimenez, J. L., Kiendler-Scharr, A., Maenhaut, W., McFiggans, G., Mentel, Th. F., Monod, A., Prévôt, A. S. H., Seinfeld, J. H., Surratt, J. D., Szmigielski, R., and Wildt, J.: The formation, properties and impact of secondary organic aerosol: current and emerging issues, Atmos. Chem. Phys., 9, 5155-5236, doi:10.5194/acp-95155-2009, 2009.

Han, Y. M., Han, Z. W., Cao J. J., Chow, J. C., Watson, J. G., An, Z. S., Liu, S. X., and Zhang, R. J.: Distribution and origin of carbonaceous aerosol over a rural high-mountain lake area, Northern China and its transport significance, Atmos. Environ., 42, 2405-2414, doi:10.1016/j.atmosenv.2007.12.020, 2008.

Han, Z., Zhang, R., Wang, Q., Wang, W., Cao, J., and Xu, J.: Regional modeling of organic aerosols over China in summertime, J. Geophys. Res., 113, D11202, doi:10.1029/2007JD009436, 2008.

Hand, J. L., Copeland, S. A., Day, D. E., Dillner, A. M., Indresand, H., Malm, W. C., McDade, C. E., Moore, C. T. Jr., Pitchford, M. L., Schichtel, B. A., and Watson, J. G.: IMPROVE (Interagency Monitoring of Protected Visual Environments): Spatial and seasonal patterns and temporal variability of haze and its constituents in the United States, Report V, CIRA Report ISSN: 0737-5352-87, Colo. State Univ., Fort Collins, 2011.

Henze, D. K. and Seinfeld, J. H.: Global secondary organic aerosol from isoprene oxidation, Geophys. Res. Lett., 33, L09812, doi:10.1029/2006GL025976, 2006.

Henze, D. K., Seinfeld, J. H., Ng, N. L., Kroll, J. H., Fu, T.-M., Jacob, D. J., and Heald, C. L.: Global modeling of secondary organic aerosol formation from aromatic hydrocarbons: highvs. low-yield pathways, Atmos. Chem. Phys., 8, 2405-2420, doi:10.5194/acp-8-2405-2008, 2008.

Huang, X., Li, M., Li, J., and Song, Y.: A high resolution emission inventory of crop burning in field in China based on MODIS thermal anomalies/fire products, Atmos. Environ., 50, 9-15, doi:10.1016/j.atmosenv.2012.01.017, 2012.

Jeong, C.-H., Hopke, P. K., Kim, E., and Lee, D.-W.: The comparison between thermal-optical transmittance elemental carbon and aethalometer black carbon measured at multiple monitoring sites, Atmos. Environ., 38, 5193-5204, doi:10.1016/j.atmosenv.2004.02.065, 2004.

Jimenez, J. L., Canagaratna, M. R., Donahue, N. M., Prevot, A. S. H., Zhang, Q., Kroll, J. H., DeCarlo, P. F., Allan, J. D., Coe, H., Ng, N. L., Aiken, A. C., Docherty, K. S., Ulbrich, I. M., Grieshop, A. P., Robinson, A. L., Duplissy, J., Smith, J. D., Wilson, K. R., Lanz, V. A., Hueglin, C., Sun, Y. L., Tian, J., Laaksonen, A., Raatikainen, T., Rautiainen, J., Vaattovaara, P., Ehn, M., Kulmala, M., Tomlinson, J. M., Collins, D. R., Cubison, M. J., Dunlea, E. J., Huffman, J. A., Onasch, T. B., Alfarra, M. R., Williams, P. I., Bower, K., Kondo, Y., Schneider, J., Drewnick, 
F., Borrmann, S., Weimer, S., Demerjian, K., Salcedo, D., Cottrell, L., Griffin, R., Takami, A., Miyoshi, T., Hatakeyama, S., Shimono, A., Sun, J. Y., Zhang, Y. M., Dzepina, K., Kimmel, J. R., Sueper, D., Jayne, J. T., Herndon, S. C., Trimborn, A. M., Williams, L. R., Wood, E. C., Middlebrook, A. M., Kolb, C. E., Baltensperger, U., and Worsnop, D. R.: Evolution of Organic Aerosols in the Atmosphere, Science, 326, 1525-1529, doi:10.1126/science.1180353, 2009.

Kondo, Y., Oshima, N., Kajino, M., Mikami, R., Moteki, N., Takegawa, N., Verma, R., Kajii, Y., Kato, S., and Takami, A.: Emissions of black carbon in East Asia estimated from observations at a remote site in the East China Sea, J. Geophys. Res., 116, D16201, doi:10.1029/2011JD015637, 2011.

Lamarque, J.-F., Bond, T. C., Eyring, V., Granier, C., Heil, A., Klimont, Z., Lee, D., Liousse, C., Mieville, A., Owen, B., Schultz, M. G., Shindell, D., Smith, S. J., Stehfest, E., Van Aardenne, J., Cooper, O. R., Kainuma, M., Mahowald, N., McConnell, J. R., Naik, V., Riahi, K., and van Vuuren, D. P.: Historical (1850-2000) gridded anthropogenic and biomass burning emissions of reactive gases and aerosols: methodology and application, Atmos. Chem. Phys., 10, 7017-7039, doi:10.5194/acp10-7017-2010, 2010.

Lei, Y., Zhang, Q., He, K. B., and Streets, D. G.: Primary anthropogenic aerosol emission trends for China, 1990-2005, Atmos. Chem. Phys., 11, 931-954, doi:10.5194/acp-11-931-2011, 2011. Liao, H., Henze, D. K., Seinfeld, J. H., Wu, S., and Mickley, L. J.: Biogenic secondary organic aerosol over the United States: comparison of climatological simulations with observations, J. Geophys. Res., 112, D06201, doi:10.1029/2006JD007813, 2007.

Liggio, J., Li, S.-M., and McLaren, R.: Reactive uptake of glyoxal by particulate matter, J. Geophys. Res., 110, D10304, doi:10.1029/2004JD005113, 2005.

Liu, H., Jacob, D. J., Bey, I., and Yantosca, R. M.: Constraints from $210 \mathrm{~Pb}$ and $7 \mathrm{Be}$ on wet deposition and transport in a global threedimensional chemical tracer model driven by assimilated meteorological fields, J. Geophys. Res., 106, 12109-12128, 2001.

Liu, H., Zhang, L., and Wu, J.: A modeling study of the climate effects of sulfate and carbonaceous aerosols over China, Adv. Atmos. Sci., 27, 1276-1288, doi:10.1007/s00376-010-9188-y, 2010.

Lu, Z., Zhang, Q., and Streets, D. G.: Sulfur dioxide and primary carbonaceous aerosol emissions in China and India, 1996-2010, Atmos. Chem. Phys., 11, 9839-9864, doi:10.5194/acp-11-98392011, 2011.

Malm, W. C., Sisler, J. F., Huffman, D., Eldred, R. A., and Cahill, T. A.: Spatial and seasonal trends in particle concentration and optical extinction in the United States, J. Geophys. Res., 99, 13471370, doi:10.1029/93JD02916, 1994.

Mari, C., Jacob, D. J., and Bechtold, P.: Transport and scavenging of soluble gases in a deep convective cloud, J. Geophys. Res., 105, 22255-22267, 2000.

Matsui, H., Koike, M., Kondo, Y., Takegawa, N., Kita, K., Miyazaki, Y., Hu, M., Chang, S.-Y., Blake, D. R., Fast, J. D., Zaveri, R. A., Streets, D. G., Zhang, Q., and Zhu, T.: Spatial and temporal variations of aerosols around Beijing in summer 2006: Model evaluation and source apportionment, J. Geophys. Res., 114, D00G13, doi:10.1029/2008JD010906, 2009.

Myriokefalitakis, S., Vrekoussis, M., Tsigaridis, K., Wittrock, F., Richter, A., Brühl, C., Volkamer, R., Burrows, J. P., and Kanaki- dou, M.: The influence of natural and anthropogenic secondary sources on the glyoxal global distribution, Atmos. Chem. Phys., 8, 4965-4981, doi:10.5194/acp-8-4965-2008, 2008.

Odum, J. R., Hoffmann, T., Bowman, F., Collins, D., Flagan, R. C., and Seinfeld, J. H.: Gas/particle partitioning and secondary organic aerosol yields, Environ. Sci. Tech., 30, 2580-2585, 1996.

Ohara, T., Akimoto, H., Kurokawa, J., Horii, N., Yamaji, K., Yan, X., and Hayasaka, T.: An Asian emission inventory of anthropogenic emission sources for the period 1980-2020, Atmos. Chem. Phys., 7, 4419-4444, doi:10.5194/acp-7-4419-2007, 2007.

Pankow, J. F.: An absorption model of gas/particle partitioning of organic compounds in the atmosphere, Atmos. Environ., 28, 185-188, 1994a.

Pankow, J. F.: An absorption model of gas/particle partitioning involved in the formation of secondary organic aerosol, Atmos. Environ., 28, 189-193, 1994b.

Park, R. J., Jacob, D. J., Chin, M., and Martin, R. V.: Sources of carbonaceous aerosols over the United States and implications for natural visibility, J. Geophys. Res., 108, 4355, doi:10.1029/2002JD003190, 2003.

Park, R. J., Jacob, D. J., Palmer, P. I., Clarke, A. D., Weber, R. J., Zondlo, M. A., Eisele, F. L., Bandy, A. R., Thornton, D. C., Sachse, G. W., and Bond, T. C.: Export efficiency of black carbon aerosol in continental outflow: global implications, J. Geophys. Res., 110, D11205, doi:10.1029/2004JD005432, 2005.

Pye, H. O. T. and Seinfeld, J. H.: A global perspective on aerosol from low-volatility organic compounds, Atmos. Chem. Phys., 10, 4377-4401, doi:10.5194/acp-10-4377-2010, 2010.

Qin, Y. and Xie, S. D.: Spatial and temporal variation of anthropogenic black carbon emissions in China for the period 1980-2009, Atmos. Chem. Phys. Discuss., 11, 32877-32920, doi:10.5194/acpd-11-32877-2011, 2011.

Qu, W. J., Zhang, X. Y., Arimoto, R., Wang, D., Wang, Y. Q., Yan, L. W., and Li, Y.: Chemical composition of the background aerosol at two sites in southwestern and northwestern China: potential influences of regional transport, Tellus, 60B, 657-673, doi:10.1111/j.1600-0889.2008.00342.x, 2008.

Robinson, A. L., Donahue, N. M., Shrivastava, M. K., Weitkamp, E. A., Sage, A. M., Grieshop, A. P., Lane, T. E., Pierce, J. R., and Pandis, S. N.: Rethinking organic aerosols: Semivolatile emissions and photochemical aging, Science, 315, 1259-1262, doi:10.1126/science.1133061, 2007.

Sareen, N., Schwier, A. N., Shapiro, E. L., Mitroo, D., and McNeill, V. F.: Secondary organic material formed by methylglyoxal in aqueous aerosol mimics, Atmos. Chem. Phys., 10, 997-1016, doi:10.5194/acp-10-997-2010, 2010.

Song, Y., Liu, B., Miao, W., Chang, D., and Zhang, Y.: Spatiotemporal variation in non-agricultural open fire emissions in China from 2000 to 2007, Global Biogeochem. Cy., 23, GB2008, doi:10.1029/2008GB03344, 2009.

Song, Y., Chang, D., Liu, B., Miao, W., Zhu, L., and Zhang, Y.: A new emission inventory for nonagricultural open fires in Asia from 2000 to 2009, Environ. Res. Lett., 5, 014014, doi:10.1088/1748-9326/5/1/014014, 2010.

Streets, D. G., Gupta, S., Waldhoff, S. T., Wang, M. Q., Bond, T. C., and Bo, Y.: Black carbon emissions in China, Atmos. Environ., 35, 4281-4296, 2001.

Streets, D. G., Bond, T. C., Carmichael, G. R., Fernandes, S. D., Fu, 
Q., He, D., Klimont, Z., Nelson, S. M., Tsai, N. Y., Wang, M. Q., Woo, J.-H., and Yarber, K. F.: An inventory of gaseous and primary aerosol emissions in Asia in the year 2000, J. Geophys. Res., 108, 8809, doi:10.1029/2002JD003093, 2003a.

Streets, D. G., Yarber, K. F., Woo, J.-H., and Carmichael, G. R.: Biomass burning in Asia: Annual and seasonal estimates and atmospheric emissions, Global Biogeochem. Cy., 17, 1099, doi:10.1029/2003GB002040, 2003b.

Turpin, B. J. and Lim, H. J.: Species contributions to PM2.5 mass concentrations: Revisiting common assumptions for estimating organic mass, Aerosol. Sci. Tech., 35, 602-610, 2001.

van der Werf, G. R., Randerson, J. T., Giglio, L., Collatz, G. J., Kasibhatla, P. S., and Arellano Jr., A. F.: Interannual variability in global biomass burning emissions from 1997 to 2004, Atmos. Chem. Phys., 6, 3423-3441, doi:10.5194/acp-6-3423-2006, 2006.

van der Werf, G. R., Randerson, J. T., Giglio, L., Collatz, G. J., Mu, M., Kasibhatla, P. S., Morton, D. C., DeFries, R. S., Jin, Y., and van Leeuwen, T. T.: Global fire emissions and the contribution of deforestation, savanna, forest, agricultural, and peat fires (19972009), Atmos. Chem. Phys., 10, 11707-11735, doi:10.5194/acp10-11707-2010, 2010.

Volkamer, R., San Martini, F., Molina, L. T., Salcedo, D., Jimenez, J. L., and Molina, M. J.: A missing sink for gas-phase glyoxal in Mexico City: Formation of secondary organic aerosol, Geophys. Res. Lett., 34, L19807, doi:10.1029/2007GL030752, 2007.

Volkamer, R., Ziemann, P. J., and Molina, M. J.: Secondary Organic Aerosol Formation from Acetylene $\left(\mathrm{C}_{2} \mathrm{H}_{2}\right)$ : seed effect on SOA yields due to organic photochemistry in the aerosol aqueous phase, Atmos. Chem. Phys., 9, 1907-1928, doi:10.5194/acp9-1907-2009, 2009.

Wang, X. M., Wu, Z. Y., and Liang, G. X.: WRF/CHEM modeling of impacts of weather conditions modified by Urban expansion on secondary organic aerosol formation over Pearl River Delta, Particuology, 7, 384-391, doi:10.1016/j.partic.2009.04.007, 2009.

Wang, Y., Jacob, D. J., and Logan, J. A.: Global simulation of tropospheric $\mathrm{O}_{3}-\mathrm{NO}_{\mathrm{x}}$-hydrocarbon chemistry: 1. Model formulation, J. Geophys. Res., 103, 10713-10726, 1998.

Wang, Y., Hao, J., McElroy, M. B., Munger, J. W., Ma, H., Chen, D., and Nielsen, C. P.: Ozone air quality during the 2008 Beijing Olympics: effectiveness of emission restrictions, Atmos. Chem. Phys., 9, 5237-5251, doi:10.5194/acp-9-5237-2009, 2009.

Wang, Y. X., McElroy, M. B., Jacob, D. J., and Yantosca, R. M.: A nested grid formulation for chemical transport over Asia: applications to CO, J. Geophys. Res., 109, D22307, doi:10.1029/2004jd005237, 2004.
Wesely, M. L.: Parameterization of surface resistance to gaseous dry deposition in regional-scale numerical models, Atmos. Environ., 23, 1293-1304, 1989.

Yang, S., He, H., Lu, S., Chen, D., and Zhu, J.: Quantification of crop residue burning in the field and its influence on ambient air quality in Suqian, China, Atmos. Environ., 42, 1961-1969, doi:10.1016/j.atmosenv.2007.12.007, 2008.

Yttri, K. E., Aas, W., Bjerke, A., Cape, J. N., Cavalli, F., Ceburnis, D., Dye, C., Emblico, L., Facchini, M. C., Forster, C., Hanssen, J. E., Hansson, H. C., Jennings, S. G., Maenhaut, W., Putaud, J. P., and Tørseth, K.: Elemental and organic carbon in $\mathrm{PM}_{10}$ : a one year measurement campaign within the European Monitoring and Evaluation Programme EMEP, Atmos. Chem. Phys., 7, 5711-5725, doi:10.5194/acp-7-5711-2007, 2007.

Zhao, J., Levitt, N. P., Zhang, R., and Chen, J.: Heteorogeneous reactions of methylglyoxal in acidic media: implications for secondary organic aerosol formation, Environ. Sci. Technol., 40, 7682-7687, doi:10.1021/es060610k, 2006.

Zhang, Q., Streets, D. G., He, K., and Klimont, Z.: Major componenets of China's anthropogenic primary particulate emissions, Environ. Res. Lett., 2, 045027, doi:10.1088/17489326/2/4/045027, 2007.

Zhang, Q., Streets, D. G., Carmichael, G. R., He, K. B., Huo, H., Kannari, A., Klimont, Z., Park, I. S., Reddy, S., Fu, J. S., Chen, D., Duan, L., Lei, Y., Wang, L. T., and Yao, Z. L.: Asian emissions in 2006 for the NASA INTEX-B mission, Atmos. Chem. Phys., 9, 5131-5153, doi:10.5194/acp-9-5131-2009, 2009.

Zhang, T., Claeys, M., Cachier, H., Dong, S., Wang, W., Maenhaut, W., and Liu, X.: Identification and estimation of the biomass burning contribution to Beijing aerosol using levoglucosan as a molecular marker, Atmos. Environ., 42, 7013-7021, doi:10.1016/j.atmosenv.2008.04.050, 2008.

Zhang, X. Y., Wang, Y. Q., Wang, D., Gong, S. L., Arimoto, R., Mao, L. J., and Li, J.: Characterization and sources of regionalscale transported carbonaceous and dust aerosols from different pathways in costal and sandy land areas of China, J. Geophys. Res., 110, D15301, doi:10.1029/2004JD005457, 2005.

Zhang, X. Y., Wang, Y. Q., Zhang, X. C., Guo, W., Gong, S. L., Zhao, P., and Jin, J. L.: Carbonaceous aerosol composition over various regions of China during 2006, J. Geophys. Res., 113, D14111, doi:10.1029/2007JD009525, 2008.

Zhou, X., Gao, J., Wang, T., Wu, W., and Wang, W. Measurement of black carbon aerosols near two Chinese megacities and the implications for improving emission inventories, Atmos. Environ., 43, 3918-3924, doi:10.1016/j.atmosenv.2009.04.062, 2009. 